\title{
The Interface Between Fresh and Salt Groundwater: A Numerical Study
}

\author{
J. R. Chan Hong, $†$ C. J. van Dulun
}

Faculty of Mathematics and Informatics, Delft University of Technology, PO Box 356, 2600 AJ Delft, The Netherlands

D. HILHORST

Laboratoire d'Analyse Numérique, CNRS et Université de Paris-Sud, 91405 Orsay, France

AND

J. VAN KESTER

Delft Hydraulics Laboratory, PO Box 177, $2600 \mathrm{MH}$ Delft, The Netherlands

[Received 8 May 1987 and in revised form 23 May 1988]

In this paper the two-dimensional flow of fresh and salt water through a homogeneous aquifer is considered. The two fluids are assumed to be separated by a sharp interface. They differ only in their specific weight. This difference induces a flow in the aquifer which in turn causes a motion of the interface.

We present a mathematical formulation of this problem which consists of a Poisson equation for the stream function coupled to a time evolution equation for the moving interface. The equation for the stream function is solved by means of a finite-element method while a predictor-corrector method (the $S^{\alpha, \beta}$ scheme) is used for the discretization of the equation for the interface.

\section{Introduction}

IN THIS paper we present a model which describes the two-dimensional motion of fresh and salt water through a horizontal aquifer. The fresh and salt water have different specific weights, denoted by $\gamma_{\mathrm{f}}$ and $\gamma_{\mathrm{s}}\left(\gamma_{\mathrm{f}}<\gamma_{\mathrm{s}}\right)$, respectively. As is frequently done in hydrology (for example see Bear $[1,2]$ ) it is assumed here that the fluids do not mix and are separated by an abrupt interface. The difference in specific weight induces a flow and thus a displacement of the fluids and the corresponding interface. Our interest here is in the evolution in time of this interface. This leads to the following rescaled problem:

$$
\left.\begin{array}{ll}
-\Delta \psi(x, z, t)=\frac{\partial}{\partial x}[\mathrm{H}(u(x, t)-z)] & \text { with }(x, z, t) \in \Omega \times \mathbb{R}^{+}, \\
\psi(x, z, t)=0 & \text { for }(x, z, t) \in \partial \Omega \times \mathbb{R}^{+}, \\
\frac{\partial u}{\partial t}(x, t)=\frac{\partial}{\partial x}[\psi(x, u(x, t), t] & \text { with }(x, t) \in I \times \mathbb{R}^{+}, \\
u(x, 0)=u_{0}(x) & \text { for } x \in I .
\end{array}\right\} \mathrm{P}
$$

† Present address: Laboratoire National d'Hydraulique, 6 quai Watier 78401 Chatou, France. 
Here $x$ and $z$ denote the horizontal and vertical space coordinates, respectively, and $t$ denotes time. Further, $I=(-1,1)$ or $I=\mathbb{R}, \Omega=I \times(0,1)$, and $\mathrm{H}$ is the Heaviside function: $\mathrm{H}(s)=1$ for $s>0$ and $\mathrm{H}(s)=0$ for $s \leqslant 0$. The initial value $u_{0}$ is assumed to be sufficiently smooth with $u_{0} \in[0,1]$ and, if $I=\mathbb{R}$, there exist numbers $-\infty<a_{1}<a_{2}<\infty$ such that

$$
\left.\begin{array}{ll}
u_{0}(x)=0 & \text { for } x \leqslant a_{1} \\
u_{0}(x) \in(0,1) & \text { for } a_{1}<x<a_{2}, \\
u_{0}(x)=1 & \text { for } x \geqslant a_{2} .
\end{array}\right\}
$$

The function $\psi$ denotes the stream function of the flow and $u$ represents the height of the fresh-salt water interface, $0 \leqslant u \leqslant 1$. At points where $u=0$ (bottom of aquifer) only fresh water is present and at points where $u=1$ (top of aquifer) only salt water is present. A full derivation of Problem $\mathbf{P}$ is given in section 2.

In section 3 we consider a simplified model which arises when a Dupuit assumption with respect to the flow is being made. Then the height $u$ of the interface satisfies a degenerate parabolic equation. We recall some analytical results about the generalized solutions of this simplified problem. The derivation of this equation, which is due to de Josselin de Jong [7], is based on the assumption that the horizontal component of the specific discharge in each fluid is constant over the height (see Fig. 3.1). Generally it is believed that this assumption holds for relatively flat interfaces. One of the purposes of this paper is to show that this is indeed the case and that the interface need not be very flat. We also show (numerically) that a solution $u$ of Problem $P$ has a large-time behaviour which is similar to the large-time behaviour of the generalized solutions of the parabolic equation. More precisely, our computations show that when $I=(-1,1), u$ converges to the constant

$$
\frac{1}{2} \int_{I} u_{0}(x) \mathrm{d} x
$$

and when $I=\mathbb{R}, u$ converges to a similarity solution of the simplified problem.

We describe the numerical method in section 4 . The equation for the stream function is solved by means of a finite-element method. The equation for the interface, when considered separately, is hyperbolic; the $S^{\alpha, \beta}$ scheme introduced by Lerat \& Peyret [14] is used for its discretization. An essential problem is to calculate as precisely as possible the $x$-coordinates $S_{1}(t)$ and $S_{2}(t)$ of the points where the interface reaches the bottom, $z=0$, and the top, $z=1$, of the aquifer. We do this by also discretizing the differential equations for $S_{1}$ and $S_{2}$ and calculating $u$ only between $S_{1}$ and $S_{2}$. Similar techniques have been used by DiBenedetto \& Hoff [9] and Hoff [13] for the discretization of the porous-media equation. The numerical results are presented in section 5. Part of the computations shown there are obtained by using a fixed triangle distribution throughout the entire flow domain. Tests are performed with and without using the extra equations for $S_{1}$ and $S_{2}$. The results are virtually the same.

A number of calculations are carried out using a triangularization of the flow domain which varies in time. This is done with the help of an automatic mesh 
generator, allowing the mesh to vary at each time step in such a way that the discretized interface coincides with sides of triangles. In this way only values of the stream function $\psi$ at mesh points are necessary in the computations. Therefore larger triangles can be used in this case. The numerical results clearly exhibit the asymptotic behaviour described in section 3 .

Certain one-phase problems are treated by related methods; in particular, Rasmussen \& Salhani [16] use a Crank-Nicolson procedure to solve the interface equation in a case where it is coupled with the Laplace equation. Duchon \& Robert $[10,11]$ consider Problem $P$ in $\Omega=\mathbb{R}^{2}$ and obtain existence and uniqueness results in a scale of Banach spaces. Chan Hong and Hilhorst $[5,6]$ prove the local existence and uniqueness of the solution of Problem $P$ in the case when $\Omega$ is the strip $\mathbb{R} \times(0,1)$ and $0<u_{0}<1$.

In the engineering literature, several authors propose finite-element models for interface problems in groundwater flow, for example, Wilson \& Da Costa [23] and Verruijt [20]. However, their work is based on the Dupuit approximation with respect to the flow. This leads to a different set of differential equations.

\section{The model}

In this section a derivation of the interface motion problem $\mathbf{P}$ is given. We consider first the problem of finding an equation for the stream function which describes the flow induced by a given variable density distribution of a fluid in a porous medium. Thereafter the case of a sharp interface is treated and an equation for the interface motion is given.

Let $h>0$. Throughout this paper we set $\Omega^{h}=I^{h} \times(0, h)$, where $I^{h}$ denotes either the interval $(-h, h)$ (bounded case) or the whole real line $\mathbb{R}$ (unbounded case).

\subsection{The Equation for the Stream Function}

Consider the 2-dimensional flow of an incompressible fluid of variable specific weight $\gamma$ and constant viscosity $\mu$ through a homogeneous porous medium with permeability $\kappa$. Let $\Omega^{h}$ denote the flow domain of the fluid. The movement of the fluid is governed by the momentum balance equation (Darcy law)

$$
\frac{\mu}{\kappa} q+\operatorname{grad} p+\gamma e_{z}=0 \text { in } \Omega^{h}
$$

and the continuity equation (expressing the incompressibility of the fluid)

$$
\operatorname{div} \boldsymbol{q}=0 \text { in } \Omega^{h} \text {. }
$$

In these equations, $q$ and $p$ denote the specific discharge field and the pressure, respectively, and $e_{z}$ denotes the unit vector in the vertical positive $z$-direction. Since we are interested in a description of the density induced flow only, we consider at the boundary $\partial \Omega^{h}$ the no-flow condition

$$
\boldsymbol{q} \cdot \boldsymbol{v}=0 \text { on } \partial \Omega^{h}
$$


where $\boldsymbol{v}$ denotes the outward normal vector on $\partial \Omega^{h}$.

In equation (2.1), $\gamma e_{z}$ is a prescribed vector which is the driving force for the flow. Taking the curl of equation (2.1) gives

$$
\operatorname{curl} q+\frac{x}{\mu} \operatorname{curl}\left(\gamma e_{x}\right)=0 \text { in } \Omega^{h},
$$

where the curl of the vectors must be understood in the sense that curl $q:=$ $\partial q_{x} / \partial z-\partial q_{z} / \partial x$. Because the flow satisfies equation (2.2) we introduce a stream function according to

$$
\boldsymbol{q}=\operatorname{curl} \psi=\left(-\frac{\partial \psi}{\partial z}, \frac{\partial \psi}{\partial x}\right)
$$

Inserting this into equation (2.4) yields the problem

$$
\left.\begin{array}{ll}
-\Delta \psi=\frac{K}{\mu} \frac{\partial \gamma}{\partial x} & \text { in } \Omega^{h}, \\
\psi=0 & \text { on } \partial \Omega^{h},
\end{array}\right\} \mathrm{P}_{\psi}
$$

where the boundary condition follows from the no-flow condition (2.3), which implies that $\psi$ is constant on $\partial \Omega^{h}$. We normalize it here to zero.

In hydrology an important example of this situation is found in the flow of fresh and salt water in coastal aquifers $[1,2]$. When the scale of the problem is small, the fluids cannot be considered to be separated by an abrupt interface. Due to mechanical dispersion and molecular diffusion a smooth transition zone develops, see de Josselin de Jong \& van Duijn [8]. However, if the scale of the problem is sufficiently large, then the fresh and salt water are often considered to be separated by a sharp interface, Verruijt [20] or Wilson \& Da Costa [23]. In particular when $\Omega^{h}$ denotes the unbounded strip $\mathbb{R} \times(0, h)$, the model describes the flow in a horizontally unconfined and vertically confined aquifer of constant thickness $h$. For this situation we have in mind a fluid distribution in which far to the left only fresh water is present, while far to the right only salt water is present, see Fig. 2.1.

In view of these applications we assume with respect to $\gamma$ the hypotheses

(i) $\gamma \in L^{\infty}\left(\Omega^{h}\right)$, and there exist real numbers $0<\gamma_{\mathrm{f}}<\gamma_{\mathrm{s}}<\infty$ such that

$\left.\begin{array}{l}\gamma_{\mathbf{f}} \leqslant \gamma \leqslant \gamma_{\mathbf{s}} . \\ \text { (ii) } \gamma \in \mathbf{L}^{2}\left(\Omega^{h}\right)+\left\{\left(\gamma_{s}-\gamma_{f}\right) \mathbf{H}(x)+\gamma_{f}\right\} \text {, where } \mathbf{H} \text { denotes the Heaviside } \\ \text { function. }\end{array}\right\} \mathrm{H}_{r}$

Hypothesis $\mathbf{H}_{\gamma}$ (ii) ensures that $\partial \gamma / \partial x \in \mathbf{H}^{-1}\left(\Omega^{h}\right)$, see Appendix $A$, Lemma $A 1$. Then it follows from standard theory (Vo Khac Khoan [21: pp. 201, 202]) that Problem $\mathrm{P}_{\psi}$ has a unique solution $\psi \in \mathrm{H}_{0}^{1}\left(\Omega^{h}\right)$. Moreover, since $\gamma \in \mathrm{L}^{\infty}\left(\Omega^{h}\right)$, it follows from Lions \& Magenes [15: Theorem 8.1] that if $\Omega$ is bounded, $\psi \in C^{0, \alpha}\left(\bar{\Omega}^{h}\right)$ for all $\alpha \in(0,1)$; furthermore one can show that if $\Omega^{h}$ is unbounded, $\psi \in \mathrm{C}^{0, \alpha}\left(\bar{\Omega}_{R}^{h}\right)$ for all $\alpha \in(0,1)$ and for all $R>0$ where $\Omega_{R}^{h}=$ $(-R, R) \times(0, h)$.

Next we show that the solution $\psi$ of Problem $P_{\psi}$, in both the bounded and unbounded case, defines a flow field which satisfies equations (2.1) to (2.3). Since 


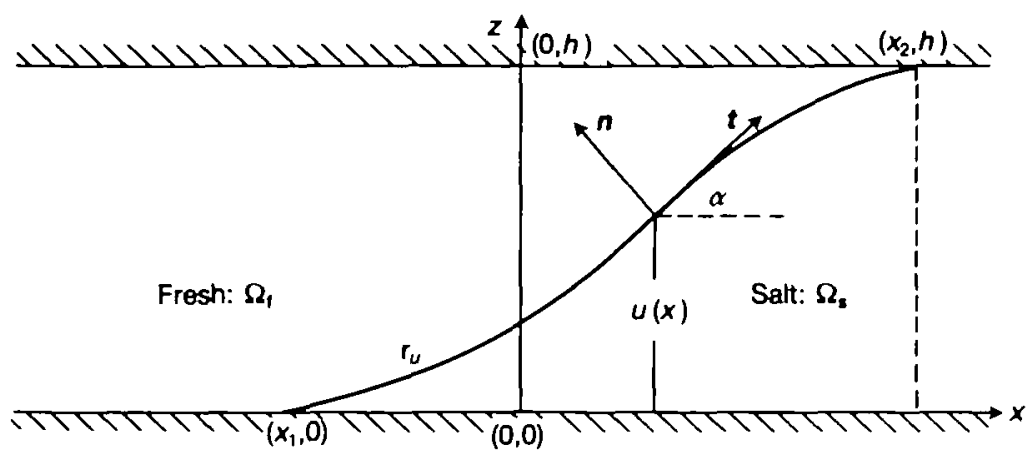

FIo. 2.1. The interface between fresh and salt groundwater in a horizontal aquifer

$\psi \in \mathrm{H}_{0}^{1}\left(\Omega^{h}\right), \quad q=$ curl $\psi \in\left\{\mathrm{L}^{2}\left(\Omega^{h}\right)\right\}^{2}$ and satisfies the no-flow condition (2.3). From (2.5) and (2.6) we have

$$
\int_{\Omega^{h}}\left(\frac{\mu}{x} q+\gamma e_{2}\right) \operatorname{curl} \phi=0 \text { for all } \phi \in \mathrm{C}_{0}^{\infty}\left(\Omega^{h}\right) .
$$

This implies by Temam [17: Propositions 1.1 and 1.2, p. 14] that there exists a function $p \in \mathrm{H}^{1}\left(\Omega^{h}\right)$ (or $p \in \mathrm{H}^{1}\left(\Omega_{R}^{h}\right)$ for all $R>0$ if $\Omega^{h}$ is unbounded) such that $q$ and $p$ satisfy Darcy's law almost everywhere in $\Omega^{h}$. Note that $p$ is uniquely defined up to a constant. Also, since $q=$ curl $\psi$, it follows by direct computation that $\langle q, \operatorname{grad} \phi\rangle=0$ for all $\phi \in C_{0}^{\infty}\left(\Omega^{h}\right)$. Thus $\operatorname{div} q=0$ in the sense of distributions.

From this point on we suppose that the scale $(h)$ of the problem is sufficiently large, so that an abrupt change in specific weight, say from fresh water with $\gamma_{\mathrm{f}}$ to salt water with $\gamma_{3}$ can be assumed. Furthermore we suppose that the height of the interface $\Gamma_{u}$, separating the fluids, can be parametrized in the form $z=u(x)$, where $u: I^{h} \rightarrow[0, h]$ is a continuous function satisfying (if $I^{h}=\mathbb{R}$ )

$$
\left.\begin{array}{ll}
u(x)=0 & \text { if } x \leqslant x_{1}, \\
u(x) \in(0, h) & \text { if } x_{1}<x<x_{2}, \\
u(x)=h & \text { if } x \geqslant x_{2},
\end{array}\right\}
$$

where $-\infty<x_{1}<x_{2}<\infty$, see Fig. 2.1. Then the specific weight throughout the flow domain is given by

$$
\gamma(x, z)=\left(\gamma_{\mathrm{s}}-\gamma_{\mathrm{f}}\right) \mathrm{H}(u(x)-z)+\gamma_{\mathrm{f}} \text { for }(x, z) \in \Omega^{h},
$$

where again $\mathrm{H}$ denotes the Heaviside function. With this expression, the equation for the stream function becomes

$$
-\Delta \psi=\Gamma \frac{\partial}{\partial x} \mathrm{H}(u(x)-z) \text { in }{\Omega^{h}}^{h}
$$

with $\Gamma=(\boldsymbol{k} / \mu)\left(\gamma_{\mathrm{s}}-\gamma_{\mathrm{f}}\right)$.

Remark 2.1. Clearly, the assumptions on the function $u$ imply that $u \in \mathrm{L}^{1}\left(I^{h}\right)+$ $\{h . H\}$. This in turn ensures that $\gamma$, defined according to (2.8), satisfies the 
hypotheses $\mathrm{H}_{\gamma}$. Thus for a given $u$, equation (2.9), with $\psi=0$ on $\partial \Omega^{h}$, has a unique solution.

Let $\Omega_{\mathrm{f}}$ denote the domain occupied by the fresh water, $\Omega_{\mathrm{s}}$ the domain occupied by the salt water; let $t$ be the tangential unit vector to $\Gamma_{u}, n$ the normal unit vector pointing into $\Omega_{\mathrm{f}}$ and $\alpha$ the angle between the positive $x$-axis and $t$, see Fig. 2.1. In the case when the function $u$ is smooth in the neighbourhood of each point $x$, where $0<u(x)<h$, we show below that the problems in terms of $q$ and $\psi$ can be written as problems on the two subdomains $\Omega_{\mathrm{s}}$ and $\Omega_{\mathrm{f}}$, with matching conditions on the interface. For convenience we use the notation $u^{\prime}$ for the $x$-derivative of $u$.

LEMMA 2.2 Let $\gamma$ be given by (2.8), in which $u$ is smooth on the set where $u \in(0, h)$. Further, let $q \in\left\{\mathrm{L}^{2}\left(\Omega^{h}\right)\right\}^{2}$ satisfy (2.1), (2.2) and (2.3) and suppose that the restrictions $q_{i}(i=\mathrm{f}, \mathrm{s})$ of $q$ to $\Omega_{i}$ are such that $q_{i} \in\left\{\mathrm{H}^{1}(\theta)\right\}^{2}$ for every open bounded set $\theta \subset \bar{\Omega}_{i}$. Then $q_{\mathrm{f}}$ and $q_{\mathrm{z}}$ satisfy

$$
\begin{aligned}
& \operatorname{div} \boldsymbol{q}_{i}=0, \quad \operatorname{curl} \boldsymbol{q}_{i}=0 \quad \text { in } \Omega_{i}, i=\mathrm{f}, \mathrm{s} ; \\
& \left(\boldsymbol{q}_{\mathrm{f}}-\boldsymbol{q}_{\mathrm{s}}\right) \cdot \boldsymbol{n}=0, \quad\left(\boldsymbol{q}_{\mathrm{f}}-\boldsymbol{q}_{\mathrm{s}}\right) \cdot \boldsymbol{t}=\Gamma \sin \alpha=\Gamma \frac{\boldsymbol{u}^{\prime}}{\left(1+\left(u^{\prime}\right)^{2}\right)^{\frac{1}{2}}} \text { on } \Gamma_{u} ; \\
& \boldsymbol{q} \cdot \boldsymbol{v}=0 \quad \text { on } \partial \Omega^{h} .
\end{aligned}
$$

Corollary 2.3 Let $\gamma$ be as in Lemma 2.2. Further, let $\psi \in \mathrm{H}_{0}^{1}\left(\Omega^{h}\right)$ satisfy Problem $\mathrm{P}_{\psi}$ and suppose that the restrictions $\psi_{i}(i=\mathrm{f}, \mathrm{s})$ of $\psi$ to $\Omega_{i}$ are such that $\psi_{i} \in \mathrm{H}^{2}(\theta)$ for every open bounded set $\theta \subset \bar{\Omega}_{i}$. Then $\psi_{\mathrm{f}}$ and $\psi_{\mathrm{s}}$ satisfy

$$
\begin{aligned}
& -\Delta \psi_{i}=0 \text { in } \Omega_{i}, i=\mathrm{f}, \mathrm{s} ; \\
& \psi_{\mathrm{f}}=\psi_{\mathrm{s}}, \quad \frac{\partial \psi_{\mathrm{f}}}{\partial n}-\frac{\partial \psi_{\mathrm{s}}}{\partial n}=-\Gamma \sin \alpha=-\Gamma \frac{u^{\prime}}{\left(1+\left(u^{\prime}\right)^{2}\right)^{\frac{1}{2}}} \text { on } \Gamma_{u} ; \\
& \psi=0 \text { on } \partial \Omega^{h} .
\end{aligned}
$$

Proof. Corollary 2.3 follows from Lemma 2.2 by substituting $\boldsymbol{q}=$ curl $\psi$.

Remark 2.4. This corollary shows that $\partial \psi / \partial n$ is discontinuous across $\Gamma_{u}$ at each point where $\Gamma_{u}$ has a non-zero slope. This will appear clearly in the numerical results.

Proof of Lemma 2.2. From

$$
\langle\operatorname{div} q, \phi\rangle=-\int_{\alpha^{\phi}} q \operatorname{grad} \phi=0 \text { for all } \phi \in \mathrm{C}_{0}^{\infty}\left(\Omega^{h}\right),
$$

it follows that $\operatorname{div} \boldsymbol{q}_{i}=0$ almost everywhere in $\Omega_{i}(i=\mathrm{f}, \mathrm{s})$ and thus that

$$
\int_{\Gamma_{s}}\left(q_{\mathrm{f}}-q_{\mathrm{s}}\right) \cdot \mathrm{n} \phi \mathrm{d} s=0 \text { for all } \phi \in \mathrm{C}_{0}^{\infty}\left(\Omega^{h}\right),
$$

which implies that $\left(\boldsymbol{q}_{\mathrm{f}}-\boldsymbol{q}_{\mathrm{s}}\right) \cdot \boldsymbol{n}=\mathbf{0}$ on $\Gamma_{u}$. Similarly, since

$$
\operatorname{curl} \boldsymbol{q}=\frac{\kappa}{\mu} \frac{\partial \gamma}{\partial x}
$$


in the sense of distributions in $\Omega^{h}$, we deduce that curl $q_{i}=0$ almost everywhere in $\Omega_{i}(i=\mathrm{f}, \mathrm{s})$ and that

$$
\int_{\Gamma_{x}}\left(q_{\mathrm{f}}-q_{\mathrm{s}}\right) \cdot t \phi \mathrm{d} s=\Gamma \int_{I^{k}} u^{\prime}(x) \phi(x, u(x)) \mathrm{d} x \text { for all } \phi \in \mathrm{C}_{0}^{\infty}\left(\Omega^{h}\right),
$$

which implies that

$$
\left(\boldsymbol{q}_{\mathrm{f}}-\boldsymbol{q}_{\mathrm{s}}\right) \cdot t=\Gamma \sin \alpha=\Gamma \frac{u^{\prime}}{\left(1+\left(u^{\prime}\right)^{2}\right)^{\frac{1}{2}}} \text { on } \Gamma_{u} .
$$

\subsection{The Interface Motion Equation; the Equations for $S_{1}$ and $S_{2}$}

Let $u=u(x, t)$ denote the height of the fresh-salt interface at a certain time $t>0$. Then the corresponding stream function can-in principle-be found by solving equation (2.9) with the boundary condition from (2.6). To stress the time dependence we write $\psi=\psi(x, z, t)$. From this the displacement of the interface is calculated as follows.

Introduce

$$
Q(x, t)=\int_{0}^{u(x, t)} q \cdot e_{x} \mathrm{~d} z
$$

as the total discharge of salt water in the horizontal, positive $x$-direction. With the definition (2.5) and the boundary condition $\psi(x, 0, t)=0$, we find that

$$
Q(x, t)=-\psi(x, u(x, t), t) \text {. }
$$

Continuity of the salt water requires, see also [7],

$$
\varepsilon \frac{\partial u}{\partial t}+\frac{\partial Q}{\partial x}=0
$$

where $\varepsilon$ is a positive constant denoting the porosity of the medium. We combine equations (2.11) and (2.12) to find the interface motion equation

$$
\varepsilon \frac{\partial u}{\partial t}(x, t)=\frac{\partial}{\partial x}\{\psi(x, u(x, t), t)\},
$$

in the region $(x, t) \in I^{h} \times \mathbb{R}^{+}$.

Note that the derivation of (2.13) is only formal because we have not enough information about the regularity of $u$ and $\psi$. Properly rescaled, see Remark 2.7, equations (2.9) and (2.13) together with boundary and initial conditions give Problem P.

We remark that in the case when $I^{h}=(-h, h)$ boundary conditions for $u$ are not needed; this comes from the fact that $\psi=0$ for $x= \pm h$ which implies that the lines $x= \pm h$ are characteristics for the equation (2.13).

Let $I^{h}=\mathbb{R}$ and let the initial distribution $u_{0}$ be of the form (2.7). Set $a_{i}:=x_{i}$ for $i=1,2$. Based on the nature of the problem we conjecture that for every $t>0$, the solution $u(\cdot, t)$ of Problem $P$ also is of the form (2.7), see Appendix B. For $i=1,2$ this leads to the existence of functions $S_{i}:[0, \infty) \rightarrow \mathbb{R}$, with $S_{i}(0)=a_{i}$ and 
$S_{1}<S_{2}$, so that the set

can be written as

$$
M:=\left\{(x, t) \in \mathbb{R} \times \mathbb{R}^{+} \mid u(x, t) \in(0,1)\right\},
$$

$$
M=\left\{(x, t) \in \mathbb{R} \times \mathbb{R}^{+} \mid S_{1}(t)<x<S_{2}(t)\right\} .
$$

Equivalently one can define, for any $t \geqslant 0$,

and

$$
S_{1}(t)=\sup \{x \in \mathbb{R} \mid u(s, t)=0 \text { for all } s \leqslant x\},
$$

$$
S_{2}(t)=\inf \{x \in \mathbb{R} \mid u(s, t)=h \text { for all } s \geqslant x\} .
$$

Next we concentrate on the differential equations for $S_{1}$ and $S_{2}$. Below we give a formal derivation concerning $S_{1}$ only. The equation for $S_{2}$ is found similarly.

First we introduce for a given position and time the salt-water weight

$$
m=\gamma_{s} \varepsilon \int_{-\infty}^{x} u(s, t) \mathrm{d} s \geqslant 0 .
$$

Conversely, expression (2.15) defines for a given $m>0$ a curve $(X(t ; m), t)$ in the $(x, t)$-plane for which the total salt-water weight on the left is constant and equal to $m$. To obtain an equation for this level curve one has to differentiate equation (2.15) with respect to $t$. This gives

$$
\left.0=\int_{-\infty}^{X(t ; m)} \frac{\partial u}{\partial t}(s, t) \mathrm{d} s+u(X / t ; m), t\right) \dot{X}(t, m),
$$

where the dot denotes differentiation with respect to time. In the integral we substitute equation (2.13). Then using the boundary condition $\psi(x, 0, t)=0$ and the fact that $u(x, t)=0$ for $x<S_{1}(t)$ we obtain the differential equation

$$
\varepsilon \dot{X}=-\frac{\psi(X, u(X, t), t)}{u(X, t)} \text { for } t>0 .
$$

At $t=0$ we have the initial condition

where $X_{0}$ is defined by

$$
X(0 ; m)=X_{0}
$$

$$
m=\gamma_{s} \varepsilon \int_{-\infty}^{x_{0}} u_{0}(s) \mathrm{d} s .
$$

By the positivity of $u$ for $X>S_{1}(t)$, we have for all $t \geqslant 0$ and for any $0<m_{1}<m_{2}<\infty$ that $X\left(t ; m_{1}\right)<X\left(t ; m_{2}\right)$ and

$$
X(t ; m) \downarrow S_{1}(t) \text { if and only if } m \downarrow 0 .
$$

Next we assume that also

$$
\dot{S}_{1}(t)=\lim _{m \downarrow 0} \dot{X}(t ; m)
$$

Then using (2.16), we have for any $t>0$

$$
\varepsilon \dot{S}_{1}(t)=-\lim _{m \downarrow 0} \frac{\psi(X(t ; m), u(X(t ; m), t), t)}{u(X(t ; m), t)}
$$


and by (2.17)

$$
\varepsilon \dot{S}_{1}(t)=-\lim _{x \backslash S_{1}(t)} \frac{\psi(x, u(x, t), t)}{u(x, t)} .
$$

For $S_{2}$ we find for any $t>0$

$$
\varepsilon \dot{S}_{2}(t)=\lim _{x \uparrow S_{2}(t)} \frac{\psi(x, u(x, t), t)}{h-u(x, t)}
$$

Equations (2.18) and (2.19) are not a part of the original problem. However, they will be used in the numerical algorithm.

Remark 2.5. With equation (2.11), one can write (2.18) as

$$
\varepsilon \dot{S}_{1}(t)=\lim _{x \downarrow S_{1}(t)} \frac{Q(x, t)}{u(x, t)} \text { for } t>0,
$$

where $Q(x, t) / u(x, t)$, for $x>S_{1}(t)$, denotes the average velocity in the horizontal direction of the salt water. Thus equation (2.18) tells us that the speed of the point $S_{1}$, the toe of the interface, is given by the average velocity of the salt water at that point.

Remark 2.6. If the height of the interface $u$ is non-decreasing on $I^{h}$, then equation (2.9) implies that $\Delta \psi \leqslant 0$. In view of the boundary conditions this means that $\psi>0$ in $\Omega^{h}$. In particular, $\psi(x, u)>0$. Based on (2.18) and (2.19) we expect that $S_{1}$ is decreasing and that $S_{2}$ is increasing. Thus the set $M$ expands in time.

Remark 2.7. Rescaling procedure. If we redefine

and

$$
x:=x / h, \quad z:=z / h, \quad u:=u / h,
$$

$$
\psi:=\psi /(\Gamma h), \quad t:=t \Gamma /(h \varepsilon),
$$

then equations (2.9) and (2.13), together with boundary and initial conditions, give Problem $P$ on the set $\Omega^{1}$. For convenience we set $\Omega:=\Omega^{1}$ and $I:=I^{1}$. The rescaled form of equations (2.18) and (2.19) is

and

$$
\dot{S}_{1}(t)=-\lim _{x \downarrow S_{1}(t)} \frac{\psi(x, u(x, t), t)}{u(x, t)},
$$

$$
\dot{S}_{2}(t)=\lim _{x \uparrow S_{2}(t)} \frac{\psi(x, u(x, t), t)}{1-u(x, t)} .
$$

Throughout the rest of this paper we work with the rescaled variables.

\section{The Dupuit approximation and some consequences}

In this section we consider a simplified form of Problem P. It arises when a Dupuit approximation with respect to the flow is made. In this approximation one assumes that the horizontal component of the velocity field is constant in each fluid and has a jump at the interface. Since $q_{x}=-\partial \psi / \partial z$ an equivalent 
assumption is that the stream function is linear in $z$ in both fluids. In hydrology one often uses this simplification, which is assumed to hold when the interfaces are not too steep. In fact our computations show that if $\partial u / \partial x \in(-1,1)$, and is not necessarily very small, this approximation is fairly reasonable (see Fig. 5.2 where $u_{0}^{\prime}=1$ and Fig. 5.7 where $\max u_{0}^{\prime}=0.50$ ). The particular form considered here is due to de Josselin de Jong [7]. It leads to a nonlinear diffusion equation for the function $u$. We recall some results about the qualitative behaviour of solutions of this equation and in particular about the large-time behaviour. The numerical simulation of solutions of Problem $\mathbf{P}$ shows many similarities, see section 5 .

\subsection{The Simplified Problem}

Our starting point is the rescaled interface motion equation of Problem P:

$$
\frac{\partial u}{\partial t}=\frac{\partial}{\partial x}\{\psi(x, u(x, t), t)\}
$$

and the observation that

or

$$
\begin{gathered}
\psi(x, u, t)=-\int_{0}^{u} q_{x}(x, z, t) \mathrm{d} z \\
\psi(x, u, t)=\int_{u}^{1} q_{x}(x, z, t) \mathrm{d} z .
\end{gathered}
$$

Using the simplifying assumption on the flow, see Fig. 3.1, the last two equations can be written as

$$
\psi(x, u, t)=-u q_{\mathrm{s}_{x}}(x, u, t)=(1-u) q_{\mathrm{f}_{x}}(x, u, t) .
$$

Further, it follows from Lemma 2.2 , with $\Gamma=1$, that

and

$$
-\left(q_{f_{x}}-q_{x_{x}}\right) \sin \alpha+\left(q_{f_{x}}-q_{x_{x}}\right) \cos \alpha=0 \text { on } \Gamma_{u}
$$

$$
\left(q_{\mathrm{t}_{x}}-q_{\mathrm{s}_{\mathrm{s}}}\right) \cos \alpha+\left(q_{\mathrm{f}_{\mathrm{z}}}-q_{\mathrm{s}_{\mathrm{s}}}\right) \sin \alpha=\sin \alpha \quad \text { on } \Gamma_{u} \text {. }
$$

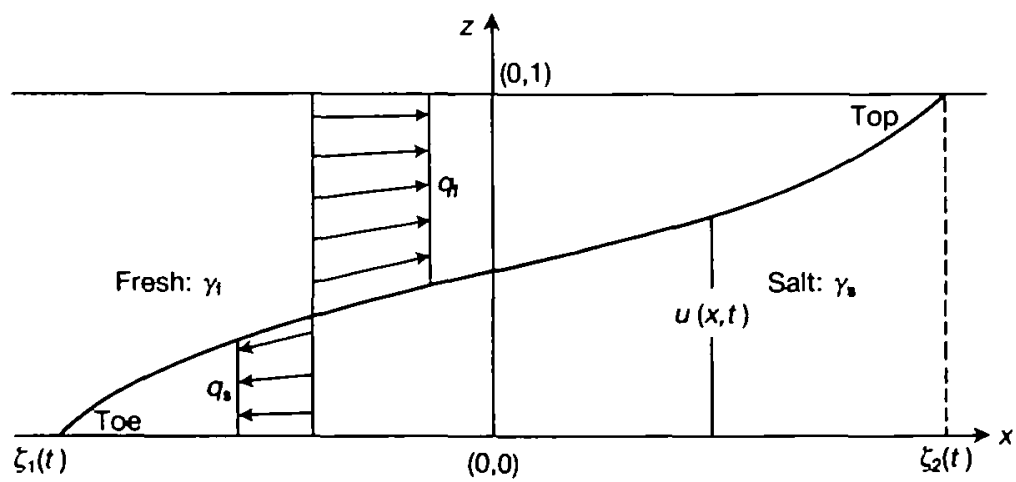

FIG. 3.1. The Dupuit approximation of the flow 
Eliminating $\left(q_{f_{z}}-q_{s_{z}}\right)$ gives

$$
q_{\mathrm{t}_{x}}-q_{\mathrm{s}_{x}}=\frac{\tan \alpha}{1+\tan ^{2} \alpha}=\frac{\partial u / \partial x}{1+(\partial u / \partial x)^{2}} .
$$

Combining (3.2) and (3.3) gives

and finally

$$
q_{t_{x}}=u \frac{\partial u / \partial x}{1+(\partial u / \partial x)^{2}} \text { and } q_{s_{x}}=-(1-u) \frac{\partial u / \partial x}{1+(\partial u / \partial x)^{2}}
$$

$$
\psi(x, u, t)=u(1-u) \frac{\partial u / \partial x}{1+(\partial u / \partial x)^{2}} .
$$

We deduce from (3.1), (3.5) and the fact that $\psi=0$ on $\partial \Omega$, that for $I=(-1,1)$, $u$ satisfies the Neumann problem

$$
\left.\begin{array}{ll}
\frac{\partial u}{\partial t}=\frac{\partial}{\partial x}\left\{u(1-u) \frac{\partial u / \partial x}{1+(\partial u / \partial x)^{2}}\right\} & \text { in } I \times \mathbb{R}^{+}, \\
u(1-u) \frac{\partial u / \partial x}{1+(\partial u / \partial x)^{2}}=0 & \text { on } \partial I \times \mathbb{R}^{+}, \\
u(\cdot, 0)=u_{0}(\cdot) & \text { on } I,
\end{array}\right\}
$$

and for $I=\mathbb{R}, u$ satisfies the Cauchy problem

$$
\left.\begin{array}{ll}
\frac{\partial u}{\partial t}=\frac{\partial}{\partial x}\left\{u(1-u) \frac{\partial u / \partial x}{1+(\partial u / \partial x)^{2}}\right\} & \text { in } I \times \mathbb{P}^{+}, \\
u(\cdot, 0)=u_{0}(\cdot) & \text { on } I .
\end{array}\right\} \mathrm{C}
$$

\subsection{Behaviour of the Solution}

Problems $\mathbf{N}$ and $\mathrm{C}$ involve differential equations which are degenerate parabolic. At points where $u \in(0,1)$ and $\partial u / \partial x \in(-1,1)$ they are parabolic, but at points where $u=0,1$ or $\partial u / \partial x=-1,1$ they degenerate: that is, the coefficient of the second-order derivative vanishes. Therefore one can in general not expect to find classical solutions. For Problems $N$ and $C$ the concept of generalized solution was introduced by van Duijn \& Hilhorst [18]. They showed that if $u_{0}$ satisfies

and

$$
u_{0} \in W^{1, \infty}(I), \quad 0 \leqslant u_{0} \leqslant 1, \quad-1 \leqslant u_{0}^{\prime} \leqslant 1 \quad \text { a.e. on } I \text {, }
$$

$$
u_{0} \in \mathrm{L}^{1}(\mathbb{R})+\{H\} \text { in the case that } I=\mathbb{R},
$$

then there exists a unique generalized solution $u$ of Problem $\mathbf{N}$ and of Problem $\mathrm{C}$. This solution is continuous on $\overline{I \times \mathbb{R}^{+}}$and it satisfies a contraction property with respect to the initial value (which implies the uniqueness). They also considered the large-time behaviour. For a solution $u$ of Problem $\mathbf{N}$ it was shown that

$$
u(\cdot, t) \rightarrow \frac{1}{2} \int_{-1}^{+1} u_{0}(x) \mathrm{d} x \quad \text { as } t \rightarrow \infty,
$$

uniformly on $[-1,1]$. 
With respect to a solution $u$ of Problem $\mathrm{C}$ they demonstrated the convergence towards a similarity solution of the form

$$
u_{s}(x, t)=s(\eta) \text { with } \eta=x f(t),
$$

where the function $s: \mathbb{R} \rightarrow[0,1]$ is given by

$$
s(\eta)=\left\{\begin{array}{lc}
0 & -\infty<\eta \leqslant-\frac{1}{2}, \\
\eta+\frac{1}{2} & -\frac{1}{2}<\eta<+\frac{1}{2}, \\
1 & \frac{1}{2} \leqslant \eta<\infty,
\end{array}\right.
$$

and where the function $f:[0, \infty) \rightarrow \mathbb{R}$ is determined by the problem

$$
\left.\begin{array}{l}
f^{\prime}(t)=-2 \frac{f^{3}}{1+f^{2}} \quad t>0, \\
f(0)=f_{0} .
\end{array}\right\} S
$$

The solution of this initial-value problem (the explicit form is given in section 5 ) is strictly increasing if $f_{0}<0$ strictly decreasing if $f_{0}>0$. Also $f(t)=O\left(t^{-\frac{1}{2}}\right)$ as $t \rightarrow \infty$. For $f_{0} \in[-1,1]$ it induces a generalized solution of Problem $C$ which has the form of a rotating line: it rotates clockwise when $f_{0} \in(0,1]$ and counterclockwise when $f_{0} \in[-1,0)$.

The convergence result of $[18]$ is:

$$
\left\|u(\cdot, t)-u_{3}(\cdot, t)\right\|_{\mathrm{L} \cdot(\mathrm{R})}=O(f(t)) \quad \text { as } t \rightarrow \infty .
$$

Recently this result was improved by Bertsch, Esteban \& Zhang [3]. They considered a shifted similarity solution for which the 'equal mass' property holds. For $a \in \mathbb{R}$, define $u_{u_{3}}(x, t)=u_{3}(x-a, t)$. Then their result is the following. Let $a \in \mathbb{R}$ be chosen such that

$$
\int_{R}\left\{u_{0}(x)-u_{u_{0}}(x, 0)\right\} d x=0,
$$

then

$$
\left\|u(\cdot, t)-u_{\mathrm{L}}(\cdot, t)\right\|_{\mathrm{L}^{-}(\mathbf{R})}=o(f(t)) \quad \text { as } t \rightarrow \infty .
$$

The convergence towards a constant $(I=(-1,1))$ and to a rotating line $(I=R)$ is also observed numerically for the approximate solution of Problem $P$, see section 5 .

It was shown by van Duijn \& Zhang [19] for solutions of Problem C that

$$
|\partial u / \partial x(\cdot, t)| \leqslant f(t) \text { a.e. on } \mathbb{R},
$$

for all $t>0$, where $f$ satisfies Problem $S$ with $f_{0}=$ ess sup $\left|u_{0}^{\prime}\right|$. Inequality (3.10) implies that $\partial u / \partial x(\cdot, t) \in(-1,1)$ for all $t>0$. Thus the degeneracy in the $x$-derivative vanishes instantaneously. As a consequence,

where

$$
u \in C^{\infty}(M) \text {, }
$$

$$
M=\left\{(x, t) \in \mathbb{R} \times \mathbb{R}^{+} \mid u(x, t) \in(0,1)\right\} .
$$

They also showed that if $u_{0}$ satisfies (1.1), then for $i=1,2$ there exist functions 
$\zeta_{i}[0, \infty) \rightarrow \mathbb{R}$, with $\zeta_{i}(0)=a_{i}$ and $(-1)^{i} \zeta_{i}$ non-decreasing on $\mathbb{R}^{+}$, such that

$$
M=\left\{(x, t) \in \mathbb{R} \times \mathbb{R}^{+} \mid \zeta_{1}(t)<x<\zeta_{2}(t)\right\} .
$$

Thus $\zeta_{1}$ and $\zeta_{2}$ are the interfaces or free boundaries related to solutions of Problem C. They separate regions where fresh water, salt water, and both are present.

In addition $\zeta_{i} \in C^{0,1}\left(\mathbb{R}^{+}\right)$and there exist numbers $t_{i}^{*} \geqslant 0$ (waiting times) such that $\zeta_{i}(t)=a_{i}$ on $\left[0, t_{i}^{*}\right]$. Further, they proved that the functions $\zeta_{i}$ are right-differentiable and that for every $t>0$ the following interface equations hold:

$$
D^{+} \xi_{1}(t)=\lim _{x \downarrow \zeta_{1}(t)} q_{s_{x}}(x, t) \text { and } D^{+} \zeta_{2}(t)=\lim _{x \uparrow \zeta_{2}(t)} q_{t_{x}}(x, t) \text {, }
$$

where $q_{\mathrm{z}_{\mathrm{x}}}$ and $q_{\mathrm{f}_{\mathrm{x}}}$ are given in (3.4).

Later it was shown by Bertsch, van Duijn, Esteban \& Zhang [4] that in fact the interfaces are continuously differentiable after the waiting times, $\zeta_{l} \in$ $C^{1}\left(t_{i}^{*}, \infty\right)$, and that equations (3.11) hold in the classical sense on $\left(t_{i}^{*}, \infty\right)$.

Observe that in view of (3.2) the interface equations (3.11) are precisely of the form of equations (2.20) and (2.21).

\section{The namerical method}

In this section we describe a numerical algorithm for solving Problem P. It is based on an explicit time integration scheme for the initial-value problem

$$
\begin{array}{ll}
\frac{\partial u}{\partial t}=\frac{\partial}{\partial x}[\psi(x, u(x, t), t)] & \text { in } I \times R^{+}, \\
u(x, 0)=u_{0}(x) & \text { on } I,
\end{array}
$$

where $I=(-1,1)$ or $I=(-R, R)$ with $R$ large enough. In practice we first have to solve the problem for the stream function $\psi$ and then to proceed with the integration in time.

\subsection{Discretization of the Problem for $\psi$}

Let $u^{n}(x)$ be the interface at time $t^{n}$. In order to determine the stream function $\psi$, we have to solve the problem

$$
\left.\begin{array}{ll}
-\Delta \psi=\frac{\partial}{\partial x}\left\{H\left(u^{n}(x)-z\right)\right\} & (x, z) \in \Omega, \\
\psi=0 & \text { on } \partial \Omega,
\end{array}\right\} \mathrm{P}_{\psi}^{n}
$$

where $\Omega=I \times(0,1)$. In the case when $I=(-R, R)$ it follows from Appendix A, Lemma A.2, that if $R$ is large enough, this problem is a good approximation for the problem in the strip $\mathbb{R} \times(0,1)$.

With the purpose of solving Problem $P_{\psi}^{n}$ by means of the finite-element method, we rewrite it in the following form. 
Find $\psi \in H_{0}^{1}(\Omega)$ such that

$$
\int_{\Omega} \operatorname{grad} \psi \operatorname{grad} v=-\int_{\Omega} \mathrm{H}\left(u^{n}(x)-z\right) \frac{\partial v}{\partial x} \text { for all } v \in \mathrm{H}_{0}^{1}(\Omega) .
$$

Let $\mathscr{T}_{h}$ be a triangularization of $\overline{\boldsymbol{\Omega}}$. Using the finite-element method with piecewise linear basis functions, we obtain the following discretized problem.

Find $\psi_{h} \in V_{h}$ satisfying

where

$$
\int_{\Omega} \operatorname{grad} \psi_{h} \operatorname{grad} v_{h}=-\int_{\Omega} \mathrm{H}\left(u_{h}^{n}(x)-z\right) \frac{\partial v_{h}}{\partial x} \text { for all } v_{h} \in V_{h},
$$

$$
V_{h}=\left\{v_{h} \in C(\bar{\Omega}) \mid \text { for all } K \in \mathscr{T}_{h}, v_{h} \text { is linear on } K \text { and } v_{h}=0 \text { on } \partial \Omega\right\},
$$

and where

$$
u_{h}^{n} \text { is a piecewise linear approximation of } u^{n} \text {. }
$$

Let $\left\{\phi_{i}\right\}, i=1, \ldots, \mathcal{N}$, be the piecewise linear basis functions (they take the value one at one node and vanish at all other nodes). Then Problem (4.3) is equivalent to the linear system

$$
\sum_{i=1}^{\mathcal{N}} \psi_{i} \int_{\mathcal{Q}} \operatorname{grad} \phi_{i} \operatorname{grad} \phi_{j}=-\int_{\mathcal{Q}} \mathrm{H}\left(u_{h}^{n}(x)-z\right) \frac{\partial \phi_{j}}{\partial x}
$$

for all $j=1, \ldots, \mathcal{N}$, where the constants $\psi_{i}, i=1, \ldots, \mathcal{N}$, are defined by

$$
\psi_{h}(x, z)=\sum_{i=1}^{\mathcal{N}} \psi_{i} \phi_{i}(x, z), \quad x, z \in \Omega .
$$

Two variants of the method have been used, with two different triangularizations of $\bar{\Omega}$.

\section{a. The fixed mesh method}

For some of the numerical tests, we have used a fixed uniform mesh, as shown in Fig. 4.1. The intervals $[-R, R]$ and $[0,1]$ are regularly subdivided, the mesh points having the coordinates

$$
\left\{\left(x_{i}=-R+i h, z_{j}=j k\right), i=0, \ldots, N, j=0, \ldots, M\right\}
$$

with $h=2 R / N$ and $k=1 / M$. To avoid the technical difficulties induced by the jump of the Heaviside function at the interface we discretize in fact the following problem

$$
\left.\begin{array}{ll}
-\Delta \psi=\frac{\partial}{\partial x}\left\{\mathrm{H}^{\varepsilon}\left(u^{n}(x)-z\right)\right\} & (x, z) \in \Omega, \\
\psi=0 & \text { on } \partial \Omega,
\end{array}\right\} \mathrm{P}_{\psi}^{\varepsilon, n}
$$

where $\mathrm{H}^{\varepsilon}(s)=\phi(s / \varepsilon)$ and $\phi \in \mathrm{C}(\mathbb{R}), \phi$ is non-decreasing, $\lim _{s \rightarrow-\infty} \phi(s)=0$, $\lim _{s \rightarrow+\infty} \phi(s)=1$. In particular, $H^{\varepsilon}$ tends to $H$ uniformly in compact subsets of $\mathbb{R} \backslash\{0\}$ as $\varepsilon \downarrow 0$. Furthermore one can show that as $\varepsilon \downarrow 0$ the solution $\psi^{\varepsilon}$ of Problem $\mathrm{P}_{\phi}^{\varepsilon, n}$ converges in $\mathrm{H}_{0}^{1}(\Omega)$ to the solution $\psi$ of Problem $\mathrm{P}_{\psi}^{n}$. 


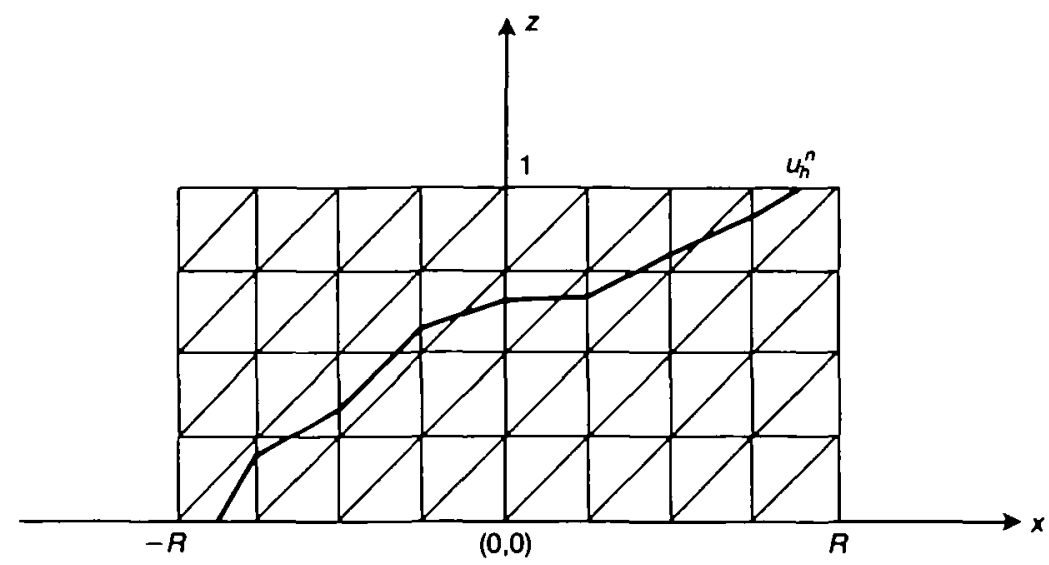

FIG. 4.1 $\mathrm{A}$ uniform mesh distribution and the interface

The computation of the right-hand side of equation (4.4) requires the evaluation of integrals of the form

$$
\int_{K} \mathrm{H}^{e}\left(u_{h}^{n}(x)-z\right) \frac{\partial p}{\partial x},
$$

where $p$ is one of the three shape functions associated with $K$. In order to calculate these integrals with sufficient precision, we use the seven-point numerical-integration formula

$$
\begin{aligned}
& \int_{K} f= \\
& \quad 2 \text { meas }(K)\left\{\frac{1}{40}\left(f\left(a_{1}\right)+f\left(a_{2}\right)+f\left(a_{3}\right)\right)+\frac{9}{40} f\left(a_{123}\right)+\frac{1}{15}\left(f\left(a_{12}\right)+f\left(a_{23}\right)+f\left(a_{13}\right)\right)\right\},
\end{aligned}
$$

where the points $a_{i}$ are given in Fig. 4.2. Finally the linear system (4.4) is solved by means of the SSOR-preconditioned conjugate gradient method.

The numerical tests have been performed with $h=k=\frac{1}{50}$ and the relaxation parameter $\omega=2 /\left(1+\sin ^{2}(\pi h)\right)$. We have chosen to take

$$
\mathrm{H}^{\varepsilon}(s)=1 / 2\left(\frac{s}{\varepsilon+|s|}+1\right) \text { with } \varepsilon=10^{-10} .
$$

However, a difficulty is that we have to calculate $\psi_{h}^{e}$ at points of the discretized interface in order to solve the interface equation and that these points are not in

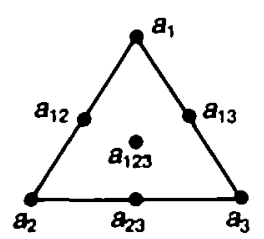

FIG. 4.2. Distribution of points for the integration formula 
general mesh points. Therefore it is necessary to use very small discretization steps $h$ and $k$. This has motivated us to construct an adaptive mesh in such a way that the discretized interface coincides with sides of triangles of $\mathscr{T}_{h}$.

\section{b. The adaptive mesh method}

The idea here is to generate a new triangularization of $\Omega$ at each time step. Let

and

$$
\Omega_{h \mathrm{f}}^{n}=\left\{(x, z) \in \Omega, z>u_{h}^{n}(x)\right\}
$$

$$
\Omega_{h \mathrm{~s}}^{n}=\left\{(x, z) \in \Omega, z<u_{h}^{n}(x)\right\} .
$$

We construct triangularizations $\mathscr{T}_{h \mathrm{f}}^{n}$ and $\mathscr{T}_{h \mathrm{~s}}^{n}$ of $\Omega_{h \mathrm{f}}^{n}$ and $\Omega_{h \mathrm{~s}}^{n}$ in such a way that the discretized interface coincides with sides of triangles of $\mathscr{S}_{h \mathrm{f}}^{n}$ and $\mathscr{G}_{h \mathrm{n}}^{n}$. In practice the meshes in $\Omega_{h \mathrm{f}}^{n}$ and in $\Omega_{h s}^{n}$ are generated by the mesh generator of the SEPRAN package of the Delft University of Technology. An example is shown in Fig. 4.3. We choose a fine mesh in the neighbourhood of the numerical interface

$$
\Gamma_{h}^{\nabla}=\bar{\Omega}_{h f}^{n} \cap \bar{\Omega}_{h s}^{n}
$$

and in particular near the endpoints of $\Gamma_{h}^{m}$ and a coarse mesh further away from $\Gamma_{\boldsymbol{h}}$. We remark that if $u^{n}$ is smooth enough, the right-hand side of the variational equality in (4.2) becomes, after integration by parts,

$$
\int_{I}\left(u^{n}\right)^{\prime}(x) v\left(x, u^{n}(x)\right) \mathrm{d} x
$$

We set $\Gamma_{h}^{n}=\bigcup_{k=1}^{\mu} \Gamma_{k}$, where the $\Gamma_{k}$ are sides of triangles. Then another form for the right-hand side of (4.4) is given by

$$
\int_{I}\left(u_{h}^{n}\right)^{\prime}(x) \phi_{j}\left(x, u_{h}^{n}(x)\right) \mathrm{d} x=\sum_{k=1}^{\mu} \int_{\Gamma_{k}} \frac{\left(u_{h}^{n}\right)^{\prime}}{\left(1+\left\{\left(u_{h}^{n}\right)^{\prime}\right\}^{2}\right)^{\frac{1}{2}}} \phi_{j} \mathrm{~d} \gamma .
$$

Here, the linear system (4.4) is solved by means of the LU decomposition.

It will be useful in section 4.2 to be able to calculate an approximation of the discharge rate $q=\operatorname{curl} \psi$ also. We set $\boldsymbol{q}_{h}=\operatorname{curl} \psi_{h}$. Since $\psi_{h}$ is piecewise linear, $q_{h}$ is constant on each triangle. On a mesh point outside $\Gamma_{h}^{n}, q_{h}$ is taken as a weighted average of the discharge rates in the triangles around that point. However, we know from Lemma 2.2 that $q$ is discontinuous across the interface; thus we must define two approximated discharge rates at mesh points on $\Gamma_{h}^{n}$ corresponding to the discretized fresh-water region and to the discretized salt-water region. On a mesh point on $\Gamma_{h}^{n}, q_{h}^{f}$ (respectively $\boldsymbol{q}_{h}^{3}$ ) is taken as a weighted average of the discharge rates on the triangles around that point which lie in $\Omega_{h \mathrm{f}}^{n}$ (respectively $\boldsymbol{\Omega}_{\mathrm{hs}}^{n}$ ).

\subsection{Discretization of the Interface Equation}

Motivated by the fact that, if $\psi$ is known, the partial differential equation in (4.1) is hyperbolic, we use the $S^{\alpha, \beta}$ explicit scheme of Lerat \& Peyret [14] with $\alpha, \beta$ 'optimal' for its discretization. There are two possibilities: either compute $u$ on the whole interval $I$ or use the extra equations (2.20), (2.21) for $S_{1}$ and $S_{2}$ and 


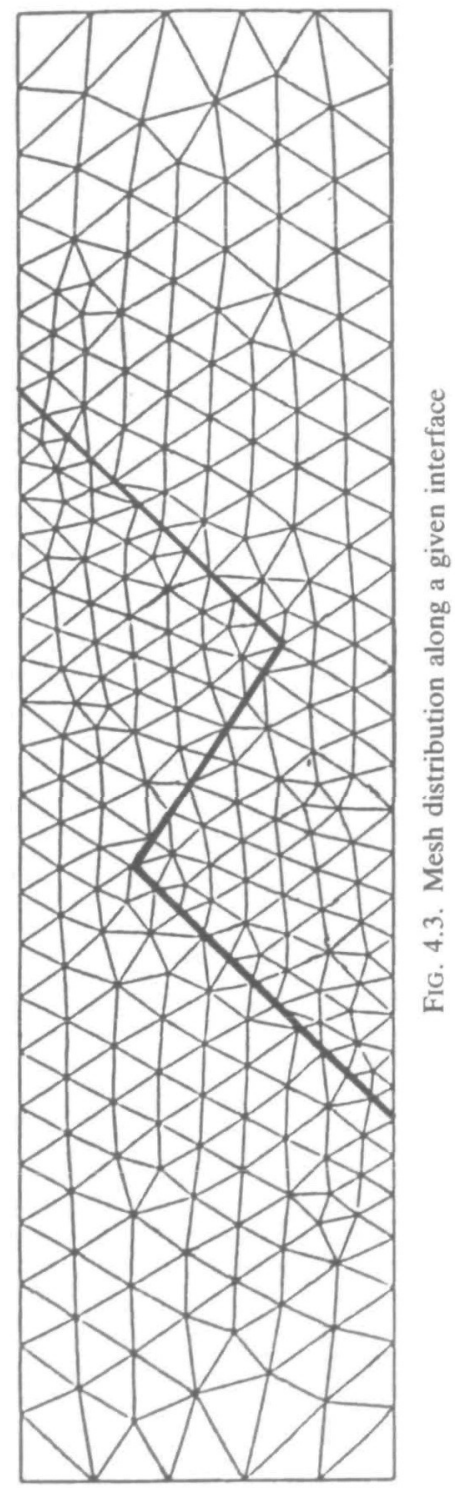


calculate $u$ only between $S_{1}$ and $S_{2}$. When programming on a fixed mesh, we can perform numerical tests in both ways and compare the results. On the other hand we always use the equations for $S_{1}$ and $S_{2}$ when calculating with an adaptive mesh.

\section{a. The $S^{\alpha, \beta}$ scheme with $\alpha, \beta$ optimal}

The $S^{\alpha, \beta}$ scheme is a second-order explicit scheme. We present it in the case when an adaptive mesh is used. Let $u_{i}^{n}$ be the approximation of $u\left(x_{i}^{n}, t^{n}\right)$. The function $u_{h}^{n}$ that we have introduced above is obtained by linear interpolation; for more details we refer to subsection 4.2.d below. Further we use the notation

$$
h_{i}^{n}=x_{i+1}^{n}-x_{i}^{n} \text { and } \Delta t^{n}=t^{n+1}-t^{n}
$$

Then the $S^{\alpha, \beta}$ scheme is given by (see [14] or [22: pp. 6-8])

$$
\begin{aligned}
\tilde{u}_{i}^{n}= & (1-\beta) u_{i}^{n}+\beta u_{i+1}^{n}+\alpha \frac{\Delta t^{n}}{h_{i}^{n}}\left\{\psi_{h}\left(x_{i+1}^{n}, u_{i+1}^{n}, t^{n}\right)-\psi_{h}\left(x_{i}^{n}, u_{i}^{n}, t^{n}\right)\right\}, \\
u_{i}^{n+1}-u_{i}^{n}= & \frac{\Delta t^{n}}{\alpha\left(h_{i}^{n}+h_{i-1}^{n}\right)}\left\{(\alpha-\beta) \psi_{h}\left(x_{i+1}^{n}, u_{i+1}^{n}, t^{n}\right)+(2 \beta-1) \psi_{h}\left(x_{i}^{n}, u_{i}^{n}, t^{n}\right)\right. \\
& \left.+(1-\alpha-\beta) \psi_{h}\left(x_{i-1}^{n}, u_{i-1}^{n}, t^{n}\right)+\tilde{\psi}_{h}\left(\tilde{x}_{i}^{n}, \tilde{u}_{i}^{n}, \tilde{t}^{n}\right)-\tilde{\psi}_{h}\left(\tilde{x}_{i-1}^{n}, \tilde{u}_{i-1}^{n}, \tilde{t}^{n}\right)\right\},
\end{aligned}
$$

where $\tilde{x}_{i}^{n}=x_{i}^{n}+\beta h_{i}^{n}, \tilde{t}^{n}=t^{n}+\alpha\left(t^{n+1}-t^{n}\right)$, the predictor term $\tilde{u}_{i}^{n}$ is an approximation of $u\left(\tilde{x}_{i}^{h}, \bar{t}^{n}\right)$ and $\psi_{h} \in V_{h}, \bar{\psi}_{h} \in \tilde{V}_{h}$ are respectively the solutions of

$$
\int_{\Omega} \operatorname{grad} \psi_{h} \operatorname{grad} v_{h}=\int_{I}\left(u_{h}^{n}\right)^{\prime}(x) v_{h}\left(x, u_{h}^{n}(x)\right) \mathrm{d} x \quad \text { for all } v_{h} \in V_{h}
$$

and

$$
\int_{Q} \operatorname{grad} \bar{\psi}_{h} \operatorname{grad} \bar{v}_{h}=\int_{l}\left(\tilde{u}_{h}^{n}\right)^{\prime}(x) \bar{v}_{h}\left(x, \tilde{u}_{h}^{n}(x)\right) \mathrm{d} x \text { for all } \bar{v}_{h} \in \bar{V}_{h},
$$

where $\tilde{u}_{h}^{n}$ and $\tilde{V}_{h}$ are the analogs of $u_{h}^{n}$ and $V_{h}$. Our choice of the parameters $\alpha=1+\sqrt{ } 5 / 2, \beta=\frac{1}{2}$ is called 'optimal' [14]. The choice $\beta=\frac{1}{2}$ ensures that the scheme (4.5), (4.6) is space centred; furthermore, when applied to Burgers' equation, this choice of the parameters minimizes the dissipative effect of the scheme.

It follows from (4.7), (4.8) that two new meshes must be generated at each time step: one for the calculation of $\psi_{h}$ at time $t^{n}$, the other for the computation of $\psi_{h}$ at time $\tau^{n}$. We remark that in the case of the fixed mesh also, the discretized version of Problem $\mathrm{P}_{\psi}^{e, n}$ is solved on a different grid for the calculation of $\psi_{h}^{\varepsilon}$; the $z$-coordinates of the mesh points remain unchanged while their $x$-coordinates become $\{(-R+i h / 2), i=0,1,3, \ldots, 2 k+1, \ldots, 2 N-1,2 N\}$.

In order to ensure the stability of the $S^{\alpha, \beta}$ scheme, we choose $\Delta t^{n}$ such that it satisfies the Courant-Friedrichs-Lewy (CFL) condition

$$
C^{n} \Delta r^{n} / h_{\min }^{n} \leqslant 1 \text {, }
$$

where $h_{\min }^{n}=\min _{i}\left\{h_{i}^{n}\right\}$, and where $C^{n}$ is an approximation of the maximum of 
$\left|\partial \psi_{h} / \partial z\right|$ on both sides on $\Gamma_{h}^{m}$. Since $q_{h x}=-\partial \psi_{h} / \partial z$, we calculate that

$$
C^{n}=\max _{\left(x_{i}^{n}, u f^{\prime}\right) \in \Gamma_{h}^{n}}\left[\max \left\{\left|q_{h x}^{f}\left(x_{i}^{n}, u_{i}^{n}, t^{n}\right)\right|,\left|q_{h x}^{s}\left(x_{i}^{n}, u_{i}^{n}, t^{n}\right)\right|\right\}\right] .
$$

In the case of the fixed mesh, we calculate $C^{n}$ as

$$
C^{n}=\max _{\substack{0<i<N \\ 0<j<M-1}}\left|\frac{\psi_{h}^{\varepsilon}\left(x_{i}, z_{j+1}, t^{n}\right)-\psi_{h}^{e}\left(x_{i}, z_{j}, t^{n}\right)}{k}\right| .
$$

\section{b. Boundary conditions}

We have already remarked that since the lines $x= \pm R$ are characteristics of the differential equation (4.1), no boundary conditions are necessary to define the analytical problem. However, we need numerical boundary conditions; we obtain them by approximating the equations on the characteristics

$$
u_{t}( \pm R, t)=\psi_{x}( \pm R, u( \pm R, t), t)
$$

Next we present some formal computations for the purpose of finding a suitable scheme, close enough to the $S^{\alpha, \beta}$ scheme used to approximate $u$ in the interior of the domain. We set

$$
\psi_{i}^{n}=\psi_{h}\left(x_{i}^{n}, u_{i}^{n}, t^{n}\right), \bar{\psi}_{i}^{n}=\bar{\psi}_{h}\left(\bar{x}_{i}^{n}, \tilde{u}_{i}^{n}, \bar{t}^{n}\right) \text { for all } i \text { and } n
$$

and deduce from (4.6) the formula

$\frac{u_{i}^{n+1}-u_{i}^{n}}{\Delta t}=$

$$
\frac{1}{2 \alpha}\left[2 \alpha \frac{\psi_{i+1}^{n}-\psi_{i-1}^{n}}{2 \Delta x}+\beta \Delta x \frac{-\psi_{i+1}^{n}+2 \psi_{i}^{n}-\psi_{i-1}^{n}}{\Delta x^{2}}-\frac{\psi_{i}^{n}-\psi_{i-1}^{n}}{\Delta x}+\frac{\psi_{i}^{n}-\psi_{i-1}^{n}}{\Delta x}\right]
$$

in which we formally let $\Delta x$ and $\Delta t$ tend to zero. We obtain the equation

$$
u_{t}=\frac{1}{2 \alpha}\left[(2 \alpha-1) \frac{\partial \psi}{\partial x}+\frac{\partial \psi}{\partial x}\right]
$$

which is completely formal but motivates us to discretize (4.10) by means of the following analog of the second-order Runge-Kutta scheme (see for instance Hildebrand [12: p. 146]):

$$
\begin{gathered}
\bar{u}_{0}=u_{0}^{n}+\alpha \Delta t^{n} \frac{\psi_{h}\left(x_{1}^{n}, u_{1}^{n}, t^{n}\right)}{h_{0}^{n}}, \\
u_{0}^{n+1}=u_{0}^{n}+\Delta t^{n}\left[\left(1-\frac{1}{2 \alpha}\right) \frac{\psi_{h}\left(x_{1}^{n}, u_{1}^{n}, t^{n}\right)}{h_{0}^{n}}+\frac{1}{2 \alpha} \frac{\psi_{h}\left(\tilde{x}_{1}^{n}, \bar{u}_{1}^{n}, \bar{t}^{n}\right)}{\bar{h}_{0}^{n}}\right]
\end{gathered}
$$

in the point $x_{0}=-R$, and similar formulae in the point $x_{N}=R$. Here we have used the fact that $\psi_{h}\left(-R, u_{0}^{n}, t^{n}\right)=\psi\left(-R, \tilde{u}_{0}^{n}, \tilde{t}^{n}\right)=0$. These boundary conditions are necessary as soon as $\Gamma_{h}^{n}$ intersects the lines $x= \pm R$. In the case of the fixed mesh and when $\Gamma_{h}^{n}$ intersects the lines $z=0$ and $z=1$, we either use them or calculate $u_{h}^{n}$ only between the approximated values of $S_{1}$ and $S_{2}$; in the case of the 
adaptive grid, we always compute $u_{h}^{n}$ only between the approximated values of $S_{1}$ and $S_{2}$.

c. Computation of $S_{1}$ and $S_{2}$

Let $S_{1}^{n}$ and $S_{2}^{n}$ be the approximated values of $S_{1}\left(t^{n}\right)$ and $S_{2}\left(t^{n}\right)$. We assume that $S_{1}^{n}>-R$ and that $S_{2}^{n}<R$ and define $k_{1}^{n}$ and $k_{2}^{n}$ by

$$
k_{1}^{n}=\min \left\{i \mid x_{1}^{n}>S_{1}^{n}\right\}, \quad k_{2}^{n}=\max \left\{i \mid x_{i}^{n}<S_{2}^{n}\right\} .
$$

The second-order Runge-Kutta method used to compute $u$ at the boundaries yields, see equations (2.20) and (2.21):

and

$$
\begin{aligned}
& \tilde{S}_{1}^{n}=S_{1}^{n}-\alpha \Delta t^{n} \frac{\psi_{h}\left(x_{k_{1}^{n}}^{n}, u_{k_{1}^{n}}^{n}, t^{n}\right)}{u_{k_{1}^{n}}^{n}}, \\
& \tilde{S}_{2}^{n}=S_{2}^{n}+\alpha \Delta t^{n} \frac{\psi_{h}\left(x_{k_{2}^{n}}^{n}, u_{k_{2}^{n}}^{n}, t^{n}\right)}{1-u_{k_{2}^{n}}^{n}},
\end{aligned}
$$

$$
\begin{aligned}
& S_{1}^{n+1}=S_{1}^{n}-\Delta t^{n}\left[\left(1-\frac{1}{2 \alpha}\right) \frac{\psi_{h}\left(x_{k_{1}^{n}}^{n}, u_{k_{1}^{n}}^{n}, t^{n}\right)}{u_{k_{j}^{n}}^{n}}+\frac{1}{2 \alpha} \frac{\psi_{h}\left(\tilde{x}_{k_{1}^{n}}^{n}, \tilde{u}_{k_{1}^{n}}^{n}, \tilde{t}^{n}\right)}{\tilde{u}_{k_{1}^{n}}^{n}}\right], \\
& S_{2}^{n+1}=S_{2}^{n}+\Delta t^{n}\left[\left(1-\frac{1}{2 \alpha}\right) \frac{\psi_{h}\left(x_{k_{2}^{n}}^{n}, u_{k_{2}^{n}}^{n}, t^{n}\right)}{1-u_{k_{2}^{n}}^{n}}+\frac{1}{2 \alpha} \frac{\psi_{h}\left(\bar{x}_{k_{2}^{n}}^{n}, \tilde{u}_{k_{2}^{n}}^{n}, t^{n}\right)}{1-\tilde{u}_{k_{2}^{n}}^{n}}\right],
\end{aligned}
$$

where

$$
\bar{k}_{1}^{n}=\min \left\{i \mid \tilde{x}_{i}^{n}>\tilde{S}_{1}^{n}\right\}, \quad \bar{k}_{2}^{n}=\max \left\{i \mid \bar{x}_{i}^{n}<\bar{S}_{2}^{n}\right\}
$$

We impose no linearized stability condition on $\Delta t^{n}$ since it would be less constraining than the CFL condition (4.9).

d. Some practical details about the computation of $u_{h}^{n}$

In the case of the fixed mesh we set

$$
u_{h}^{n+1}(x)= \begin{cases}0 & \text { for all } x \leqslant S_{1}^{n+1} \\ 1 & \text { for all } x \geqslant S_{2}^{n+1}\end{cases}
$$

and use formulae (4.5), (4.6) to calculate $u_{i}^{n+1}$ for $i \in\left\{k_{1}^{n+1}, \ldots, k_{2}^{n+1}\right\}$. We then define $u_{h}^{n+1}$ on $\left(S_{1}^{n+1}, S_{2}^{n+1}\right)$ by linear interpolation. An example is shown in Fig. 4.1.

In the case of the adaptive mesh, we define $u_{h}^{n+1}$ by (4.11) outside $\left(S_{1}^{n+1}, S_{2}^{n+1}\right)$ and calculate values $\bar{u}_{j}^{n+1}$ in previously computed mesh points by using formulae (4.5), (4.6). This yields a first discretized interface at time $t^{n+1}$

$$
\left(S_{1}^{n+1}, 0\right),\left(S_{1}^{n}, \bar{u}_{k_{1}^{*}-1}^{n+1}\right),\left(x_{k_{1}^{n}}^{n}, \bar{u}_{k_{1}^{n}}^{n+1}\right), \ldots,\left(x_{k_{2}^{n}}^{n}, \bar{u}_{k_{2}^{*}}^{n+1}\right),\left(S_{2}^{n}, \bar{u}_{k_{2}^{n}+1}^{n+1}\right),\left(S_{2}^{n+1}, 1\right) .
$$

Then we make a new distribution $\left\{x_{i}^{n+1}\right\}$ of the interval $\left(S_{1}^{n+1}, S_{2}^{n+1}\right)$ based on a new distribution of coordinates along the interface and use linear interpolation to compute $\left\{u_{i}^{n+1}\right\}$. 


\section{Numerical results}

In this section we present and discuss a number of numerical results. To give a proper interpretation one has to use the scaling formulae from Remark 2.7. In particular we have for the dimensionless time $t$, the interface height $u$ and the toe (top) position $S_{i}(i=1,2)$ :

$$
t_{\text {real }}=\frac{\varepsilon h \mu}{\kappa\left(\gamma_{\mathrm{s}}-\gamma_{\mathrm{f}}\right)} t, \quad u_{\text {real }}=h u, \quad S_{i_{\text {read }}}=h S_{i} .
$$

According to Lemma 2.2 the specific discharge field is discontinuous across the interface $\Gamma_{u}$ between the fluids. We have

$$
\left(\boldsymbol{q}_{\mathrm{f}}-\boldsymbol{q}_{\mathrm{s}}\right) \cdot \boldsymbol{n}=0, \quad\left(\boldsymbol{q}_{\mathrm{f}}-\boldsymbol{q}_{\mathrm{s}}\right) \cdot \boldsymbol{t}=\sin \alpha,
$$

at $z=u(x)$, where $\tan \alpha=u^{\prime}(x)$.

Equation (5.1) is called the shear flow and it creates the vorticity in the fluids. This follows directly if we put the results of Lemma 2.2 in the form

$$
\operatorname{div} q=0, \quad \operatorname{curl} q(r)=\int_{r_{\mathrm{s}}}\left(q_{\mathrm{f}}-q_{\mathrm{s}}\right) \cdot t \delta\left(r-r^{\prime}\right) \mathrm{d} l^{\prime},
$$

in the sense of distributions in $\Omega$, where $r=(x, z) \in \Omega, r^{\prime}=\left(x^{\prime}, u\left(x^{\prime}\right)\right)$ with $x^{\prime} \in I$ and $\delta$ is the Dirac distribution. Thus the shear flow being the driving force for the flow, it is important to approximate it accurately with our numerical method. We give below an example to show that this is indeed the case. Let

$$
u_{0}(x)= \begin{cases}0 & -1 \leqslant x \leqslant-\frac{1}{2} \\ x+\frac{1}{2} & -\frac{1}{2}<x<\frac{1}{2}, \\ 1 & \frac{1}{2} \leqslant x \leqslant 1 .\end{cases}
$$

The normal (pointing into the fresh water) and the tangential unit vector at the interface are given by

$$
n=(-\sin \alpha, \cos \alpha), \quad t=(\cos \alpha, \sin \alpha) .
$$

Then it follows from (5.1) that

or

$$
\left(q_{\mathrm{t}}-q_{\mathrm{z}}\right)_{\mathrm{x}}=\sin \alpha \cos \alpha, \quad\left(q_{\mathrm{f}}-q_{\mathrm{s}}\right)_{\mathrm{z}}=\sin ^{2} \alpha,
$$

$$
\left(\frac{\partial \psi_{\mathrm{f}}}{\partial z}-\frac{\partial \psi_{\mathrm{s}}}{\partial z}\right)=-\sin \alpha \cos \alpha, \quad\left(\frac{\partial \psi_{\mathrm{f}}}{\partial x}-\frac{\partial \psi_{\mathrm{s}}}{\partial x}\right)=\sin ^{2} \alpha
$$

In Fig. 5.1 the triangle distribution with the adaptive grid method is shown for the interface (5.2). Due to the discontinuity in the derivatives of $\psi$, the space steps were taken smaller in a neighbourhood of the interface in order to obtain a good approximation of the stream function. We used here 80 discretization points on the interface between $S_{1}$ and $S_{2}$. Figure 5.2 shows level curves of the stream function in $\Omega$ : the value of $\psi$ increases from $\psi=0$ on $\partial \Omega$ to $\psi=0.125$ in the centre point $\left(0, \frac{1}{2}\right)$ in constant steps. One observes from this picture that for a 


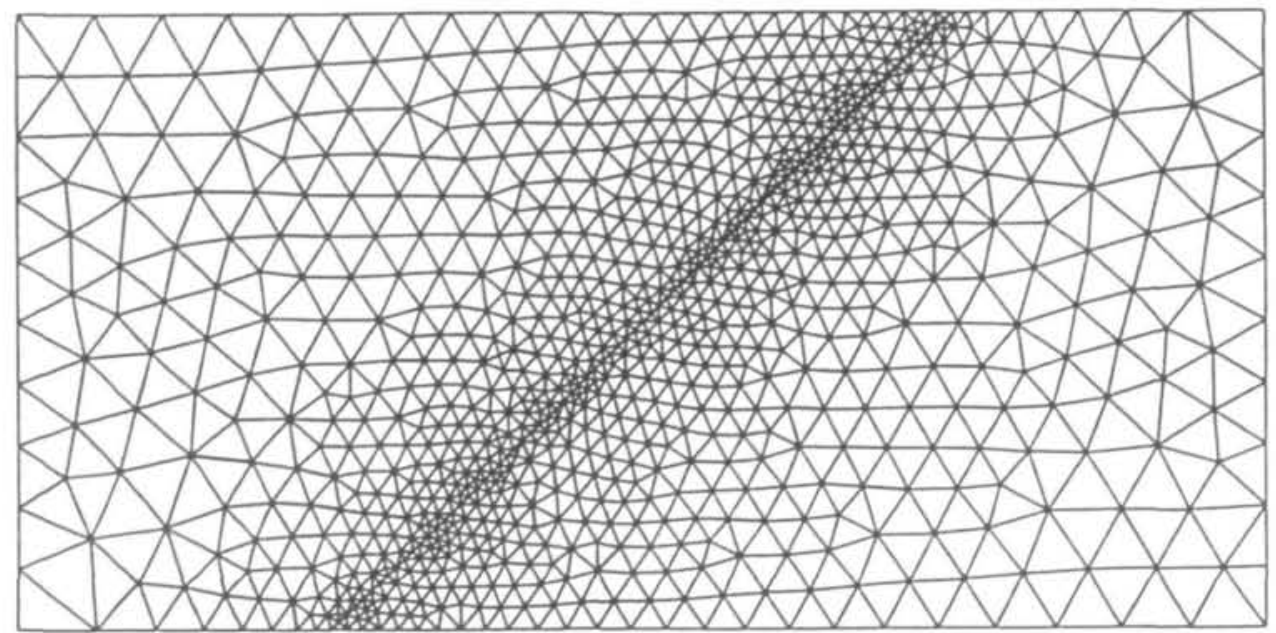

Fio. 5.1. Triangle distribution obtained with the adaptive grid procedure. The interface is given by (5.2)

neighbourhood of $x=0$, say $-0.25<x<0.25$, the Dupuit approximation is valid: for fixed $x$, the height of the level curves is nearly equidistant, showing that $\psi$ is linear in both the fluids. At the interface, the theoretical jump in the components of the specific discharge is given by, see expressions (5.3),

$$
\left[q_{x}\right]=0.50 \text { and }\left[q_{x}\right]=0 \cdot 50 \text {. }
$$

Numerically we obtained the results shown in Table 5.1.

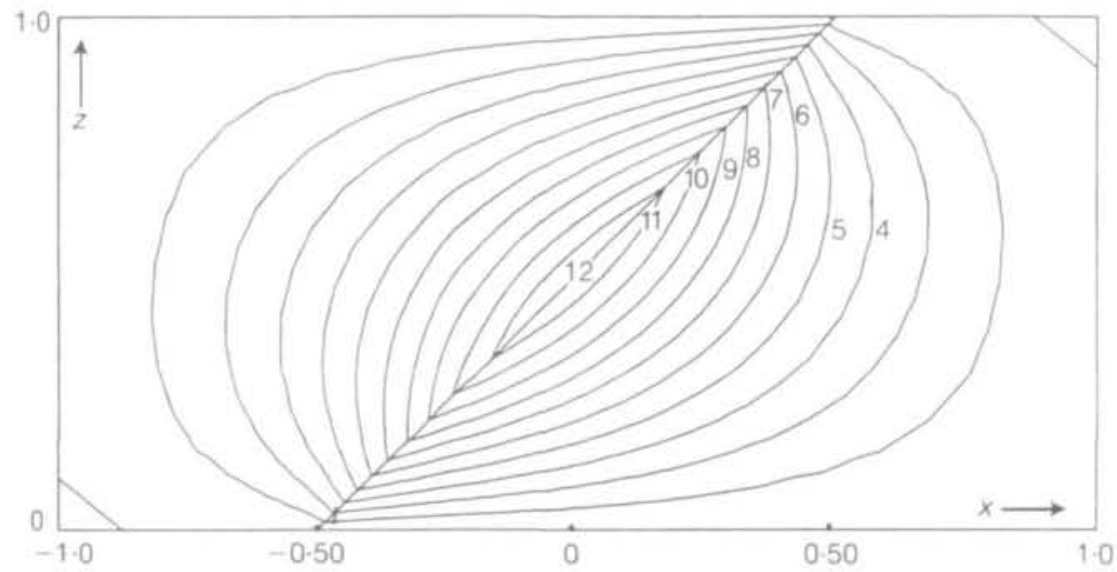

Fig. 5.2. Level curves of the stream function in $\Omega$. The value of $\psi$ increases from $\psi=0$ on $\partial \Omega$ to $\psi=0.125$ in the centre point, in constant steps 
TABle 5.1

\begin{tabular}{ccc}
\hline $\begin{array}{c}\text { Height of the points } \\
\text { on the interface }\end{array}$ & {$\left[q_{x}\right]$} & {$\left[q_{z}\right]$} \\
\hline$\frac{1}{8}$ & 0.490 & 0.476 \\
$\frac{1}{8}$ & 0.495 & 0.480 \\
$\frac{3}{8}$ & 0.485 & 0.491 \\
$\frac{4}{8}$ & 0.497 & 0.493 \\
$\frac{5}{8}$ & 0.498 & 0.493 \\
$\frac{6}{8}$ & 0.497 & 0.492 \\
$\frac{7}{8}$ & 0.499 & 0.496 \\
\hline
\end{tabular}

Next we considered the time evolution of the interface. As a test case we computed the solution of Problem $\mathrm{P}$ on $\Omega=(-1,1) \times(0,1)$ with the initial condition

$$
u_{0}(x)=\left\{\begin{array}{lr}
0, & -1 \leqslant x<-\frac{1}{2}, \\
2 x+1, & -\frac{1}{2} \leqslant x<-\frac{1}{6}, \\
-x+\frac{1}{2}, & -\frac{1}{6} \leqslant x<\frac{1}{6}, \\
2 x, & \frac{1}{6} \leqslant x<\frac{1}{2}, \\
1, & \frac{1}{2} \leqslant x \leqslant 1 .
\end{array}\right.
$$

We performed the computations with both the adaptive and the fixed grid method. Because the results differ very little we present in Figs. 5.3 to 5.6 only the adaptive mesh calculations. First we determined the stream function $\psi(x, z, t=0)$ and the corresponding velocity field $q=\operatorname{curl} \psi$. Figure 5.3 gives

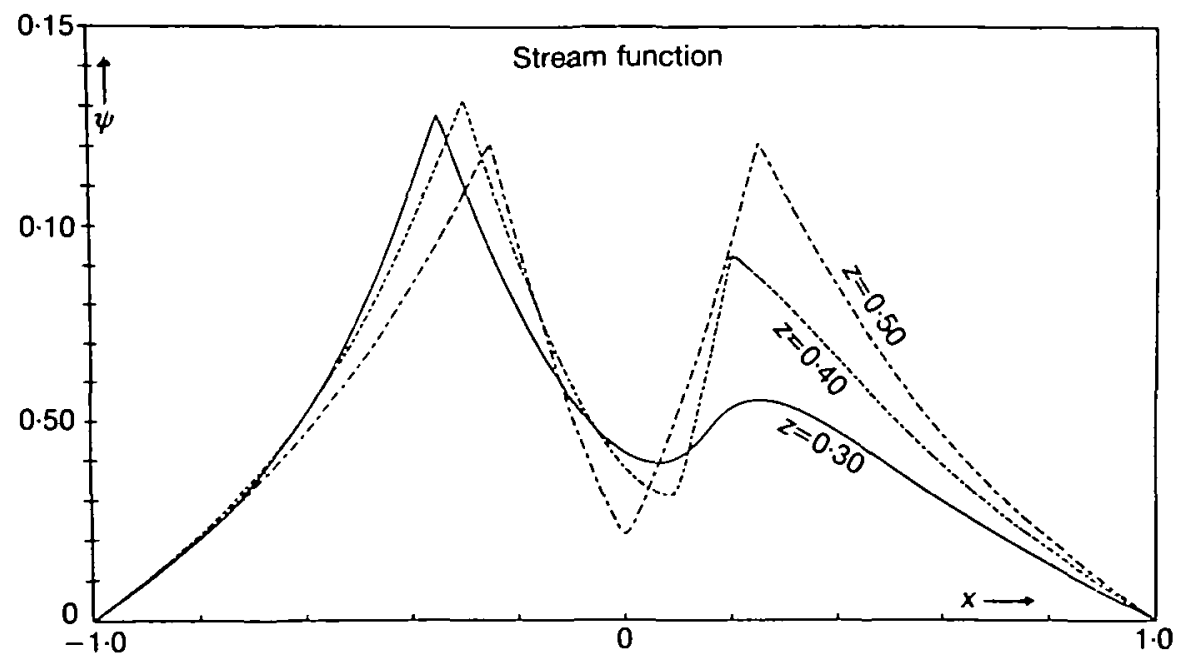

FIG. 5.3. The stream function induced by the initial distribution (5.4) 


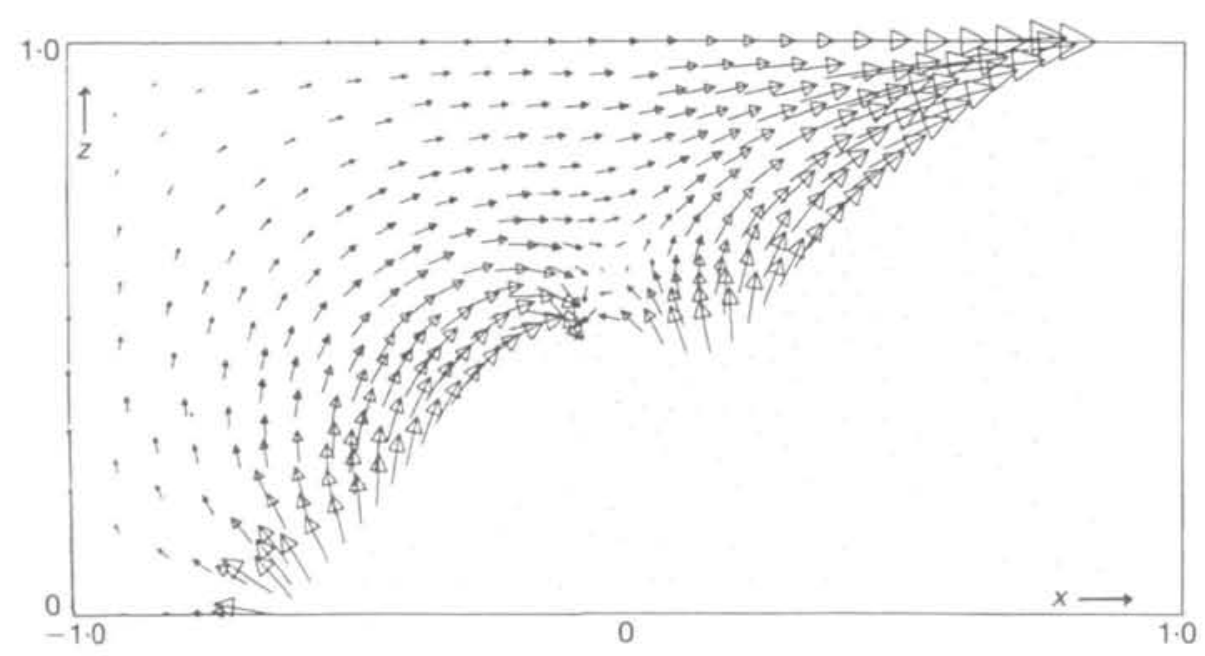

Fig. 5.4. The velocity field of the fresh water induced by the initial distribution (5.4)

$\psi(\cdot, z, t=0)$ for several values of the height $z$ and Fig. 5.4 shows the induced velocity field. Again observe the jumps in $\partial \psi / \partial x$ at the interface points.

Due to these discontinuities the space steps must be taken small (at least in a neighbourhood of the interface) in order to obtain a reasonable approximation of the stream function along $\Gamma_{u}$. With the adaptive mesh, the triangles generated near the interface are smaller than those in the far field and a triangle distribution similar to the one shown in Fig. 4.3 is used. When using the fixed, uniform mesh we take $h=k=\frac{1}{30}$.

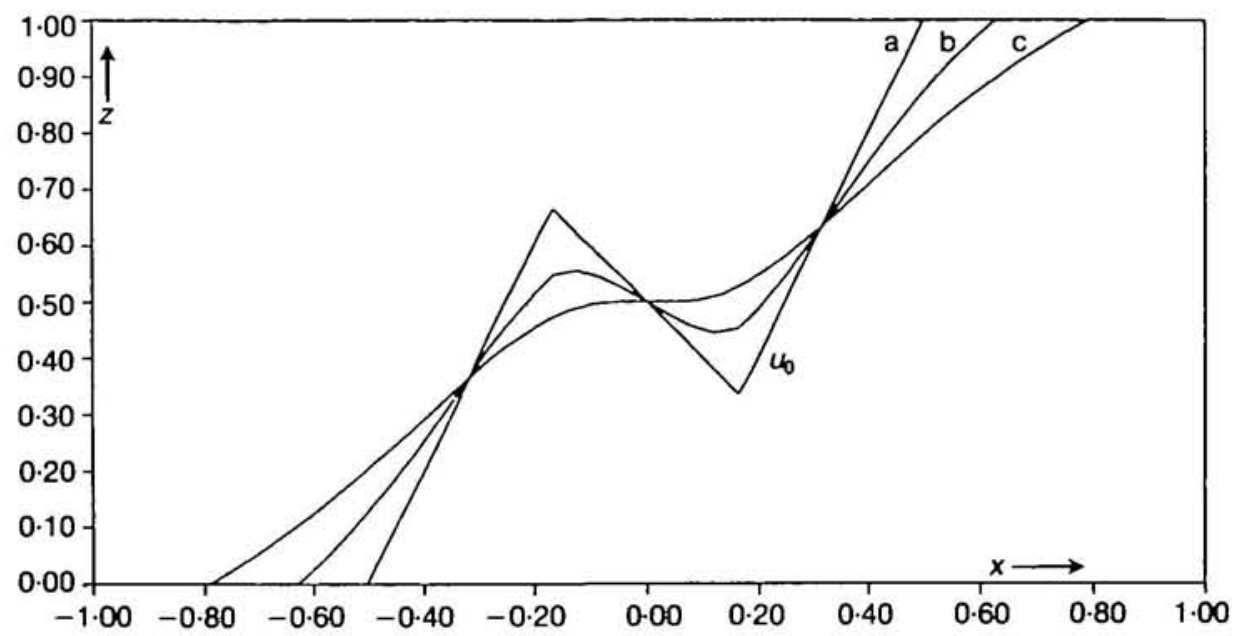

Fig. 5.5. Interfaces computed between $S_{1}$ and $S_{2}$ with the moving mesh method. The initial value (a) is given by (5.4). the interfaces are given at $t=0.14$ (b) and $t=0.38$ (c). We used here $\alpha=2.118$, $\beta=0.50$, and $\Delta r^{n}=0.5 \Delta r_{C r L}^{n}$ 


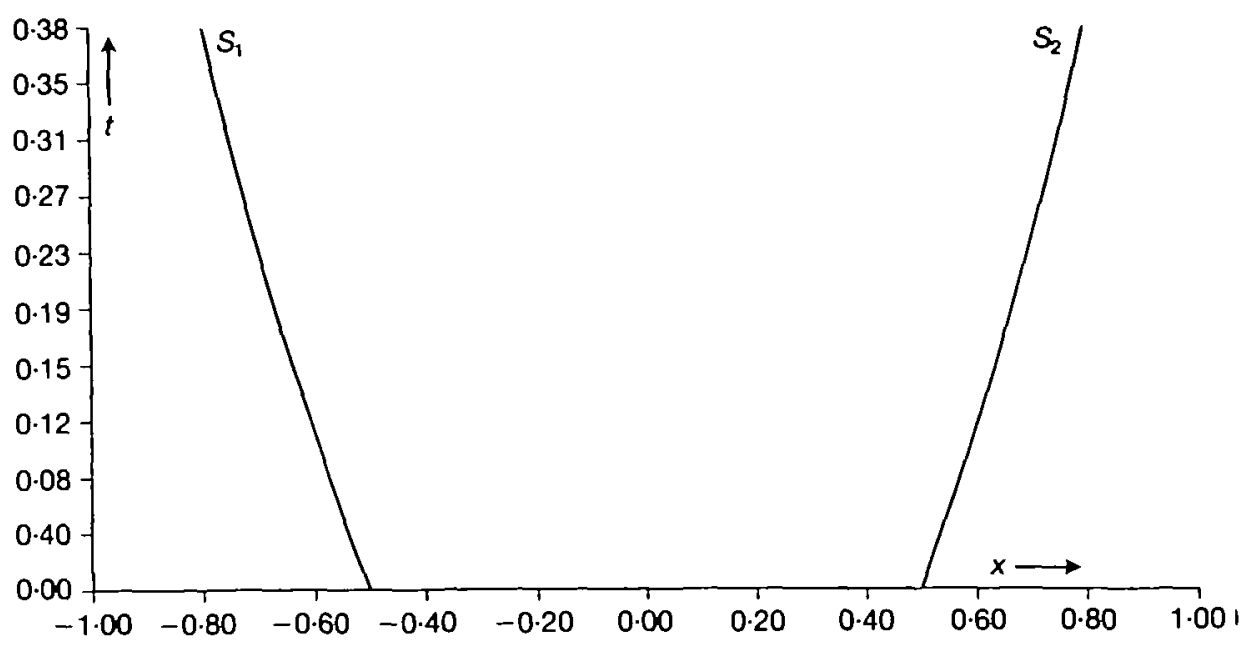

Fio. 5.6. The evolution of the corresponding free boundaries $S_{1}$ and $S_{2}$, see also Fig. 5.5

In Figs. 5.5 and 5.6 the time evolution of the solution and the corresponding interfaces $S_{1}$ and $S_{2}$ are shown. When using the moving mesh procedure the solution is computed by means of the $S^{\alpha \beta}$ scheme, with $\alpha$ and $\beta$ 'optimal', between the points $S_{1}$ and $S_{2}$. We have taken, for the time step,

$$
\Delta t^{n}=\frac{1}{2} \Delta t_{\mathrm{CF}}^{n}
$$

where $\Delta t_{\mathrm{CFL}}^{n}=h_{\min }^{n} / C^{n}$, see inequality (4.9).

Computations were also done with $\Delta t^{n}=\Delta t_{\text {CFL. }}$. Then we observed small jumps in the velocity of the toe and the top, which seem to occur at values of $t$ where the difference $\left|S_{1}\left(t^{n+1}\right)-S_{1}\left(t^{n}\right)\right|$ or $\left|S_{2}\left(t^{n+1}\right)-S_{2}\left(t^{n}\right)\right|$ is equal to the local space step: this difference then has the maximum value allowed by the CFL condition. This has motivated us to perform the numerical computations with time steps satisfying (5.5).

When one solves Problem $P$ with the fixed mesh, more grid points and consequently smaller time steps are necessary. Comparing the results with those obtained with the moving mesh we find that the differences in the positions of the curves $S_{1}$ and $S_{2}$ are less than four per cent. As stated in section 4.2.b, the interface can also be computed without the use of the differential equations for $S_{1}$ and $S_{2}$. Here again the resulting curves differ very little.

The next results concern the Dupuit approximation and the large time behaviour of solutions of Problem $\mathrm{P}$. We again solve Problem $\mathrm{P}$ on $\Omega=$ $(-1,1) \times(0,1)$ but now with the initial condition

$$
u_{0}(x)=(\tanh x+1) / 2 \text { for } x \in(-1,1) .
$$

Figures 5.7 and 5.8 give the results obtained with a fixed grid in which $k=h=\frac{1}{60}$. Figure 5.7 shows the stream function $\psi(x, \cdot, t=0)$ for various values of $x$. This picture clearly demonstrates the validity of the Dupuit assumption which says that 


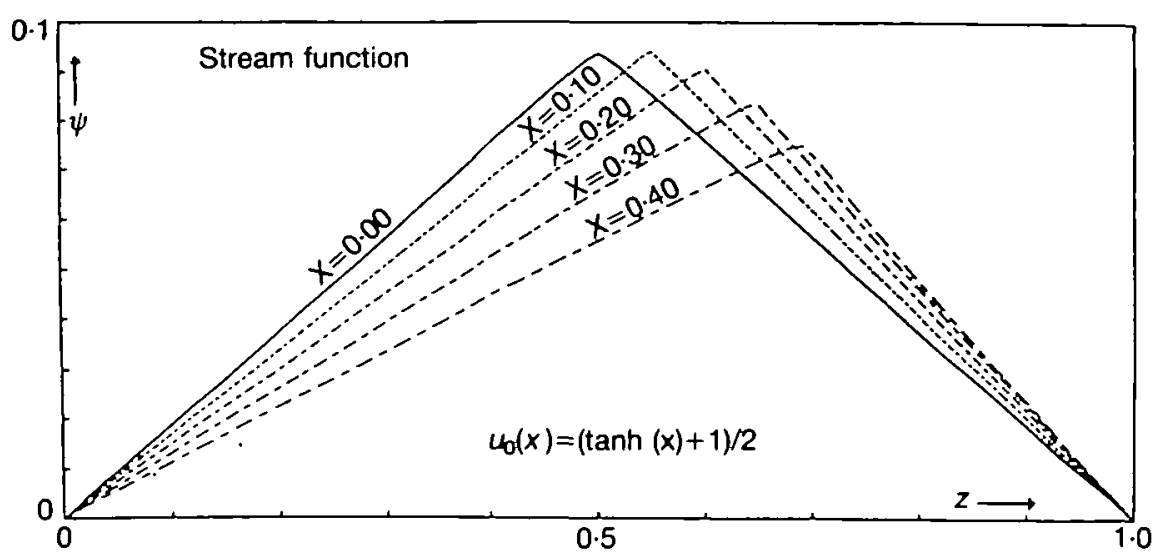

FIG. 5.7. The stream function $\psi(x, \cdot)$ for various values of $x$ induced by the initial distribution $u_{0}$. The linear behaviour of $\psi$ in $z$ clearly justifies the Dupuit approximation

the horizontal discharge component $q_{x}$ is constant in each fluid. It is implied by the fact that for fixed $x \in(-1,1)$ the stream function is linear in $z$ on each subdomain. In this case the slope of $u_{0}$ is comparatively large: it is maximal at $x=0$ where $u_{0}^{\prime}(0)=0.5$. We also remark that the jump of $\partial \psi / \partial z$ is well approximated at that point: its theoretical value is -0.40 while its numerical value is -0.38 .

Figure 5.8 demonstrates the convergence of the numerical solution towards the equilibrium position

$$
\frac{1}{2} \int_{-1}^{+1} u_{0}(x) \mathrm{d} x=0 \cdot 5
$$

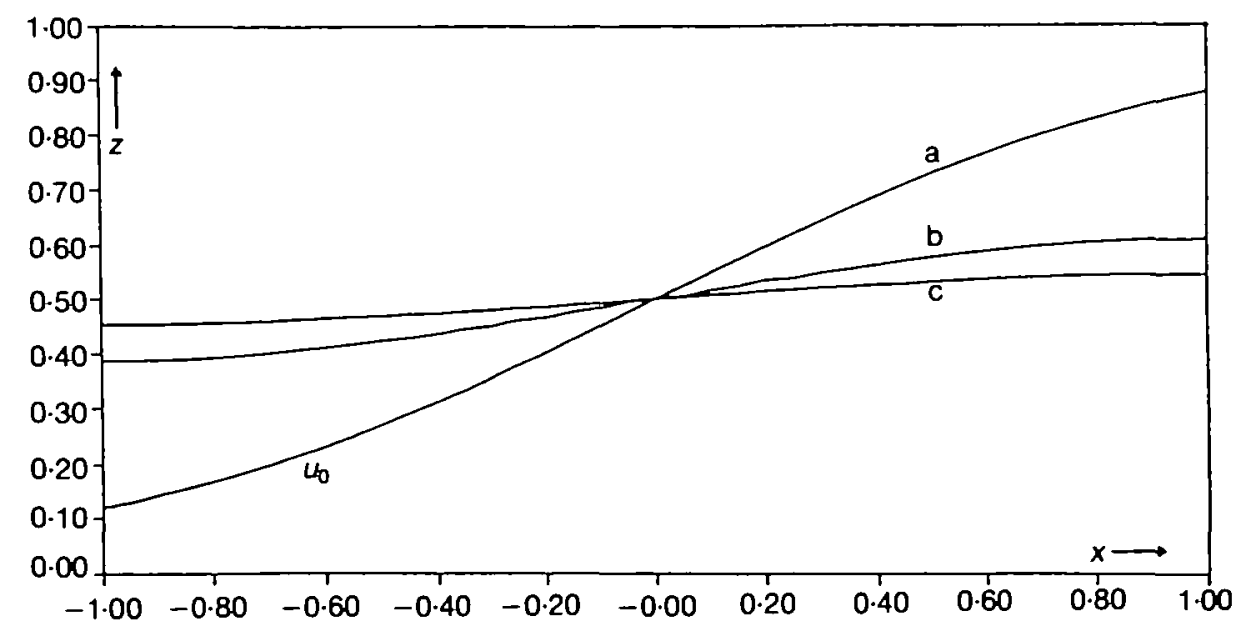

Fio. 5.8. Convergence to a constant. Interfaces computed with the fixed mesh method. The initial distribution (a) is given by (5.6). The interfaces are given at $t=2.5$ (b) and $t=4.25$ (c). The time steps are chosen to be $\Delta r^{n}=h$ 


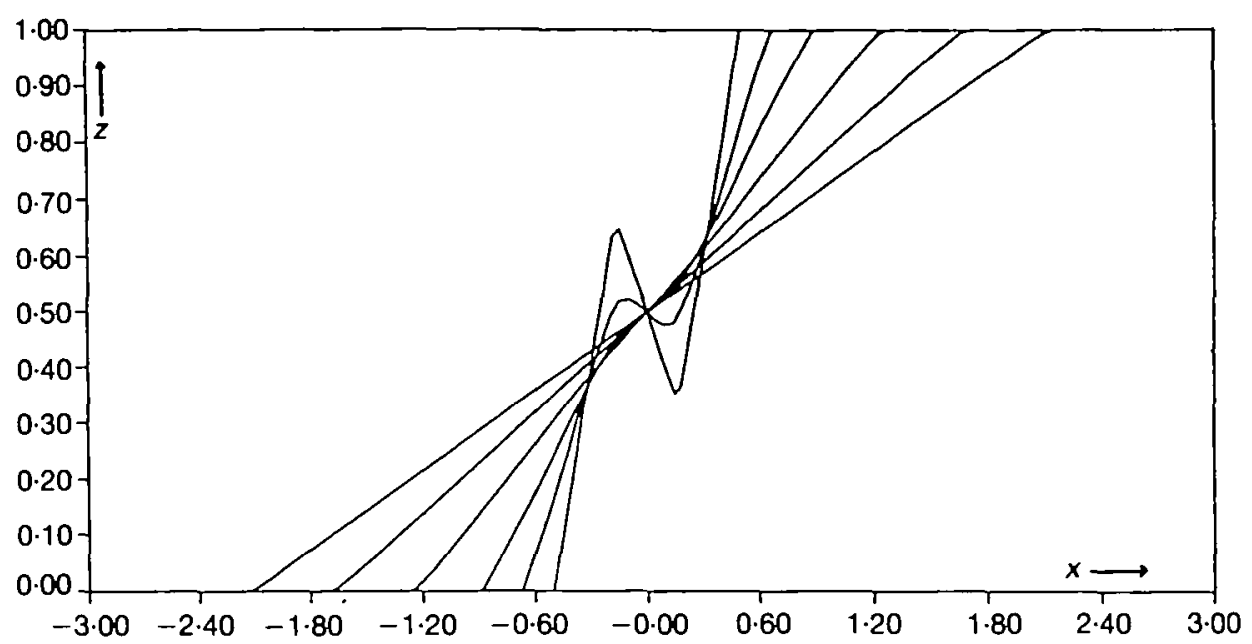

FiG. 5.9. Convergence to the similarity solution. The interfaces are computed with the moving mesh method. The initial distribution is given by (5.4). The interfaces are shown at $t=0.24 ; t=0.60$; $t=1 \cdot 43 ; t=2 \cdot 77$; and $t=4.57$

This behaviour corresponds to what we expect from the simplified (Dupuit) problem, as discussed in section 3 . When the equilibrium is approached, the velocity of the fluids becomes smaller. Therefore $\Delta t_{\mathrm{CFL}}^{n}$ becomes larger than the space step $h$. This led us to choose $\Delta t^{n}=h$. Figure 5.9 shows the large-time behaviour of the numerical solution of Problem $\mathrm{P}$ on the domain $\Omega=(-3,3) \times$ $(0,1)$. At $t=0$ we chose again the initial value (5.4) which we extended by $u_{0}=0$ to the left and by $u_{0}=1$ to the right. The computations were performed with the adaptive mesh procedure. One observes that for large $t$ the interface behaves as a rotating line. In section 3 we also encountered a rotating line as a similarity solution of the simplified Problem C. Motivated by the convergence result of that section, we compare the computed solution of Problem $\mathbf{P}$ with the similarity solution $u_{\mathbf{z}}$ for which

$$
\int_{\mathbf{R}}\left\{u_{0}(x)-u_{z}(x, 0)\right\} \mathrm{d} x=0 .
$$

By the symmetry of the initial value we can take

$$
u_{3}(x, t)=s(\eta) \text { and } \eta=x f(t)
$$

where $s$ is defined below definition (3.7) and where $f$ is the solution of Problem $S$ with $f_{0}=1$. An easy computation shows that

with

$$
f(t)=\phi^{-1}\left(2 t+\frac{1}{2}\right) \text { for } t \geqslant 0,
$$

$$
\phi(s)=\frac{1}{2} s^{-2}-\ln s \text { for } s \in(0,1] \text {. }
$$

The function $u_{z}$ defines also two interface curves in the $(x, t)$-plane: for $t \geqslant 0$ we 
TABLE 5.2

\begin{tabular}{ccc}
\hline$t$ & $S_{2}(t)$ (Computed values) & $S_{2}(t)$ \\
\hline 1.428 & 1.26 & 1.13 \\
2.770 & 1.69 & 1.56 \\
4.570 & 2.14 & 2.03 \\
\hline
\end{tabular}

have

$$
S_{1}(t)=-1 /(2 f(t)) \text { and } S_{2}(t)=+1 /(2 f(t)) \text {, }
$$

where the points $\left(\tilde{S}_{1}(t), t\right)$ correspond to $\eta=-\frac{1}{2}$ and $\left(\tilde{S}_{2}(t), t\right)$ correspond to $\eta=+\frac{1}{2}$. In Table 5.2, the values of $S_{2}$ and $\bar{S}_{2}$ are given at several times.

\section{Actnowledgements}

The authors acknowledge a number of constructive discussions with $\mathrm{Ph}$. Clement about the formulation of the problem. They also wish to thank Ch.H. Bruneau and $\mathrm{P}$. Wilders for pointing out the hyperbolic character of the problem and for suggesting the use of the $S^{\alpha, \beta}$ method. They are indebted to J. Laminie for his technical help and patient explanations. The first and third authors also wish to thank C. M. Brauner and C. Schmidt-Lainé for useful discussions when this work started.

\section{REFERENCES}

1. Bear, J. 1975 Dynamics of Fluids in Porous Media. New York: American Elsevier.

2. BeAR, J. 1979 Hydraulics of Groundwater. New York: McGraw-Hill.

3. Bertsch, M., Esteban, J. R., \& Zhang, H. 1988 On the asymptotic behaviour of the solutions of a degenerate equation in hydrology. Preprint, Delft.

4. Bertsch, M., van Duin, C. J., Esteban, J. R., \& Zhano, H. 1989 Regularity of the free boundary in a doubly degenerate parabolic equation. Communs part. diff. Eqns, to appear.

5. Chan Hong, J. R. 1987 Sur quelques problèmes à frontière libre en hydrologie. Ph.D. Thesis, Ecole Centrale de Lyon.

6. Chan Hono, J. R., \& Hilhorst, D. 1987 The interface between fresh and salt groundwater. Proceedings, International Colloquium on Free Boundary Problems: Theory and Applications, Irsee, to appear.

7. DE JOSSELIN DE JONG, G. 1981 The simultaneous flow of fresh and salt water in aquifers of large horizontal extension determined by shear flow and vortex theory. Proceedings, Euromech. 143 (eds A. Verruijt and F. B. J. Barends). Rotterdam: Balkema.

8. De Josselin De Jong, G., \& van DuiJn, C. J. 1986 Transverse dispersion from an originally sharp fresh-salt interface caused by shear flow. J. Hydrology 84, 55-79.

9. DiBenedetro, E., \& Hoff, D. 1984 An interface tracking algorithm for the porous media equation. Trans. Amer. math. Soc. 284, 463-500.

10. Duchon, J., \& Robert, R. 1985 Perturbation quasi-différentielle d'un semi-groupe régularisant dans une échelle d'espaces de Banach. C.R. Acad. Sci. Paris 301, $561-564$.

11. Duchon, J., \& Robert, R. 1986 Estimation d'opérateurs intégraux du type de Cauchy dans les Echelles d'Ovsjannikov et application. Ann. Inst. Fourier 36, 83-95.

12. Hiddebrand, F. B. 1968 Finite-Difference Equations and Simulations. Englewood Cliffs: Prentice Hall. 
13. HoFF, D. 1985 A scheme for computing solutions and interface curves for a doubly degenerate parabolic equation. SIAM J. numer. Anal. 22, 687-712.

14. Lerat, A., \& Peyret, R. 1973 Sur le choix de schémas aux différences finies du second ordre fournissant des profils de choc sans oscillation. C.R. Acad. Sci. Paris 277, 363-366.

15. Lions, J. L., \& Magenes, E. 1961 Problemi ai limiti non omogenei III. Ann. Scuola Norm Sup. Pisa 15, 41-101.

16. Rasmussen, H., \& Salhan, D. 1981 Unsteady porous flow with a free surface. IMA J. appl. Math. 27, 307-318.

17. Tемам, R. 1979 Navier-Stokes Equation, Theory and Numerical Analysis. Amsterdam: North-Holland.

18. van Duin, C. J., \& Hilmorst, D. 1987 On a doubly nonlinear diffusion equation in hydrology. Nonlinear Anal. Theory Meth. Appl. 11, 305-333.

19. van DuIN, C. J., \& Zhang, H. 1988 Regularity properties of a doubly degenerate equation in hydrology. Communs part. diff. Eqns 13, 261-319.

20. VERRUIT, A. 1985 A finite element method for interface problems in groundwater flow. Microcomputers in Engineering Applications (ed. B. A. Schrefler). New York: Wiley.

21. Vo Khac Khoan 1972 Distributions, Analyse de Fourier, Operateurs aux Dérivées Partielles, Tome II. Paris: Vuibert.

22. WiLders, P. 1983 Minimization of dispersion in difference methods for hyperbolic conservation laws. Doctoral Thesis, University of Amsterdam.

23. Wilson, J. L., \& DA-CostA, A. 1982 Finite element simulation of a salt water-fresh water interface with indirect toe tracking. Water Resour. Res. 18, 1069-1080.

\section{Appendir A}

LEMMA A.1 Let $\gamma$ satisfy the hypothesis $\mathrm{H}_{\gamma}$. Then $\partial \gamma / \partial x \in \mathrm{H}^{-1}\left(\Omega^{h}\right)$.

Proof. If $\Omega^{h}$ is bounded, the result follows immediately. If $\Omega^{h}$ is unbounded we set

$$
g(x, z):=\left(\gamma_{\mathrm{t}}-\gamma_{\mathrm{f}}\right) \mathrm{H}(x)+\gamma_{\mathrm{f}} . \quad(x, z) \in \Omega^{h} .
$$

Proving Lemma A.1 amounts to showing that $\partial g / \partial x \in \mathrm{H}^{-1}\left(\Omega^{h}\right)$. Let $\mathrm{H}_{n}$ be a smooth non-decreasing approximation of $H$ such that $0 \leqslant H_{n} \leqslant 1, H_{n}(x)=0$ for $x \leqslant-1 / n$ and $H_{n}(x)=1$ for $x \geqslant 1 / n$ and define

$$
g_{n}(x, z):=\left(\gamma_{\mathrm{s}}-\gamma_{\mathrm{f}}\right) \mathrm{H}_{n}(x)+\gamma_{\mathrm{f}} .
$$

Then $\lim _{n \rightarrow \infty}\left\|g_{n}-g\right\|_{L^{2}\left(\Omega^{h}\right)}=0$. Let $v \in \mathrm{H}_{0}^{1}\left(\Omega^{h}\right)$ be arbitrary. Denoting by $\langle\cdot, \cdot\rangle$ the duality product between $\mathrm{H}^{-1}\left(\Omega^{h}\right)$ and $\mathrm{H}_{0}^{1}\left(\Omega^{h}\right)$ we have

$$
\begin{aligned}
& \left|\left\langle\frac{\partial g_{n}}{\partial x}, v\right\rangle\right|=\left|\int_{\alpha} \frac{\partial g_{n}}{\partial x} v\right|=\left|\int_{0}^{h} \int_{-1 / n}^{1 / n} \frac{\partial g_{n}}{\partial x} v\right| \\
& =\left|-\int_{0}^{h} \int_{-1 / n}^{1 / n} g_{n} \frac{\partial v}{\partial x}+\int_{0}^{h}\left(\gamma_{s} v\left(\frac{1}{n}, z\right)-\gamma_{\mathrm{f}} v\left(-\frac{1}{n}, z\right)\right) d z\right| \\
& \leqslant\left(\frac{2 h}{n}\right)^{\frac{1}{2}} \gamma_{s}\|v\|_{H_{0}^{\prime}\left(\infty^{\infty}\right)}+\gamma_{s} V_{h}\left\{\left\|v\left(\frac{1}{n}, \cdot\right)\right\|_{L^{2}(0, h)}+\left\|v\left(-\frac{1}{n}, \cdot\right)\right\|_{L^{2}(0, h)}\right\} \\
& \leqslant C \gamma_{3}\|v\|_{\mathrm{H}_{0}^{1}\left(\boldsymbol{\sigma}^{\star}\right)} \text {. }
\end{aligned}
$$

Since, furthermore, $g_{n}-g \rightarrow 0$ in $\mathrm{L}^{2}\left(\Omega^{h}\right)$, the result follows at once. 
LEMMA A.2 Let $\Omega=\mathbb{R} \times(0,1)$ and for $R>0$ let $\Omega_{R}=(-R, R) \times(0,1)$ Further, let $\psi_{R}$ be the solution of Problem $\mathrm{P}_{\psi}$ on the domain $\Omega_{R}$ and let $\psi_{R}$ be the extension of $\psi_{R}$ on $\Omega$ with $\bar{\psi}_{R}=0$ on $\Omega \backslash \Omega_{R}$. Then

$$
\psi_{R} \rightarrow \psi \text { weakly in } H_{0}^{1}(\Omega) \text { as } R \rightarrow \infty \text {, }
$$

where $\psi$ is the solution of Problem $\mathrm{P}_{\psi}$ on $\Omega$.

Proof. By construction, $\bar{\psi}_{R} \in \mathbf{H}_{0}^{1}(\Omega)$. It satisfies

$$
\begin{aligned}
\int_{0}\left(\operatorname{grad} \psi_{R}\right)^{2} & =\int_{\boldsymbol{O}_{k}}\left(\operatorname{grad} \psi_{R}\right)^{2}=-\frac{\kappa}{\mu} \int_{\boldsymbol{O}_{R}} \gamma \frac{\partial \psi_{R}}{\partial x} \\
& =-\frac{\kappa}{\mu} \int_{\Omega} \gamma \frac{\partial \psi_{R}}{\partial x} \leqslant \frac{\kappa}{\mu}\left\|\frac{\partial \gamma}{\partial x}\right\|_{\mathbf{H}^{-1}(\boldsymbol{O})} \cdot\left\|\psi_{R}\right\|_{\mathrm{H}_{0}^{1}(\boldsymbol{\Omega})} .
\end{aligned}
$$

This yields $\left\|\tilde{\psi}_{R}\right\|_{\mathrm{H}_{0}^{1}(\Omega)} \leqslant C$. The result then easily follows.

\section{Appenth B}

In this appendix all variables are rescaled according to Remark 2.7. Here we analyse the possibility of finite or infinite speed of propagation of the toe $S_{1}$ and the top $S_{2}$ of the interface. We present a formal analysis which is based on an explicit form of $\psi$. For a given interface height $u=u(x)$ we solve the equation for the stream function on the domain $\mathbb{R} \times(0,1)$ such that $\psi(\cdot, 0)=\psi(\cdot, 1)=0$ on $\mathbb{R}$.

For convenience we suppress the time dependence. We only consider the situation near $S_{1}$. For $S_{2}$ a similar argument applies. We confine ourselves to the case where $u: \mathbb{R} \rightarrow[0,1]$ is a continuous function that behaves as a power near $S_{1}$. For simplicity we assume that

for some constant $m>1$.

$$
u(x)= \begin{cases}0 & \text { for } x \leqslant 0 \\ x^{m} & \text { for } 0<x<1 \\ 1 & \text { for } x \geqslant 1\end{cases}
$$

The stream function at the interface is given by (see [2: p. 363])

$$
\psi(x, u(x))=-\frac{1}{4 \pi} \int_{0}^{1} \ln \left\{\frac{\cosh (\bar{x}-\tilde{s})-\cos (\tilde{u}(x)-\tilde{u}(s))}{\cosh (\bar{x}-\bar{s})-\cos (\tilde{u}(x)+\tilde{u}(s))}\right\} u^{\prime}(s) \mathrm{d} s,
$$

where $\bar{x}=\pi x, \tilde{s}=\pi s$, and $\tilde{u}=\pi u$. Motivated by (2.20) we investigate

$$
\lim _{x \downarrow 0} \frac{\psi(x, u(x))}{u(x)}
$$

If this limit is finite (respectively infinite), we say that the toe moves at a finite (respectively infinite) speed. Let $v$ denote the inverse of $u$ on $(0,1)$. Then for a given $\delta \in(0,1)$ we consider

$$
\psi(v(\delta), \delta)=-\frac{1}{4 \pi} \int_{0}^{1} \ln \left\{\frac{\cosh (\tilde{v}(\delta)-\bar{v}(s))-\cos (\tilde{\delta}-\bar{s})}{\cosh (\tilde{v}(\delta)-\tilde{v}(s))-\cos (\tilde{\delta}+\bar{s})}\right\} \mathrm{d} s,
$$


and the limit

$$
\lim _{\delta \downarrow 0} \frac{\psi(v(\delta), \delta)}{\delta}
$$

By Remark 2.6, $\psi>0$ in $\Omega$. Therefore we consider only positive bounds on $\psi(v(\delta), \delta) / \delta$.

Expression (B.4) can be written as

$$
\psi(v(\delta), \delta)=-\frac{1}{4 \pi} \int_{0}^{1} \ln \left\{1-\frac{2 \sin \delta \sin \bar{s}}{\cosh (\tilde{v}(\delta)-\bar{v}(s))-\cos (\delta+\bar{s})}\right\} \mathrm{d} s .
$$

Introduce a fixed $\varepsilon \in(0,1)$ and $L>1$. Then for $\delta$ sufficiently small we have

$$
0<\delta L<\varepsilon<1 \text {. }
$$

Next we consider $\psi(v(\delta), \delta)$ as the sum of three integrals

$$
\psi=\psi_{0}+\psi_{\delta L}+\psi_{\varepsilon} \quad \text { at }(v(\delta), \delta)
$$

where $\psi_{0}$ denotes the contribution from the interval $(0, \delta L), \psi_{\delta L}$ from $(\delta L, \varepsilon)$ and $\psi_{\varepsilon}$ from $(\varepsilon, 1)$.

We start with

$$
\psi_{\varepsilon}(v(\delta), \delta)=-\frac{1}{4 \pi} \int_{\varepsilon}^{1} \ln \left\{1-\frac{2 \sin \delta \sin \tilde{s}}{\cosh (\tilde{v}(\delta)-\tilde{v}(s))-\cos (\delta+\tilde{s})}\right\} \mathrm{d} s
$$

For small $\delta$, the argument of the logarithm in the integrand is near 1 . Then it follows, using the concavity of the logarithm, that there exists a constant $C(\delta)>1$ (with $C(\delta) \downarrow 1$ as $\delta \downarrow 0$ ) such that

$$
\begin{aligned}
\int_{\varepsilon}^{1} \frac{\sin \delta \sin \bar{s}}{\cosh (\tilde{v}(\delta)-\tilde{v}(s))-\cos (\tilde{s}+\delta)} \mathrm{d} s & <2 \pi \psi_{\varepsilon}(v(\delta), \delta) \\
& <C(\delta) \int_{e}^{1} \frac{\sin \delta \sin \bar{s}}{\cosh (\tilde{v}(\delta)-\tilde{v}(s))-\cos (\tilde{s}+\delta)} \mathrm{d} s .
\end{aligned}
$$

Consequently,

$$
\lim _{\delta s \downarrow 0} \frac{\psi_{\varepsilon}(v(\delta), \delta)}{\delta}=\frac{1}{2} \int_{\varepsilon}^{1} \frac{\sin \bar{s}}{\cosh \tilde{v}(s)-\cos \bar{s}} \mathrm{~d} s<\infty .
$$

Next we consider the contribution $\psi_{0}$. For $\varepsilon$ small enough we may approximately write (putting $y=s / \delta$ )

$$
\frac{\psi_{0}(v(\delta), \delta)}{\delta}=-\frac{1}{4 \pi} \int_{0}^{L} \ln \left\{1-\frac{4 y}{(v(\delta) / \delta-v(y \delta) / \delta)^{2}+(1+y)^{2}}\right\} \mathrm{d} y .
$$

Using $v(s)=s^{p}$, with $p=1 / m<1$, this becomes

$$
\frac{\psi_{0}(v(\delta), \delta)}{\delta}=-\frac{1}{4 \pi} \int_{0}^{L} \ln \left\{1-\frac{4 y}{\delta^{2(p-1)}\left(1-y^{p}\right)^{2}+(1+y)^{2}}\right\} d y .
$$

The integrand, including the minus sign, is positive and bounded above by the integrable function $-\ln \left[1-\left(4 y /(1+y)^{2}\right)\right]$. Moreover, for $\delta \downarrow 0$ the integrand converges pointwise to zero on $(0,1) \cup(1, L)$. By the dominated convergence 
theorem we obtain

$$
\lim _{\delta \downarrow 0} \frac{\psi_{0}(v(\delta), \delta)}{\delta}=0
$$

What remains is the contribution $\psi_{\delta L}$. Again, since $\varepsilon$ is small, we write for simplicity the approximation

Since

$$
\psi_{\delta L}(v(\delta), \delta)=-\frac{1}{4 \pi} \int_{\delta L}^{\varepsilon} \ln \left\{1-\frac{4 \delta s}{(v(\delta)-v(s))^{2}+(\delta+s)^{2}}\right\} \mathrm{d} s
$$

we again have

$$
\frac{\delta s}{(v(\delta)-v(s))^{2}+(\delta+s)^{2}}<\frac{\delta}{s}<\frac{1}{L} \quad \text { for } s \in(\delta L, \varepsilon),
$$

$$
\begin{aligned}
\int_{\delta L}^{\varepsilon} \frac{s}{(v(\delta)-v(s))^{2}+(\delta+s)^{2}} \mathrm{~d} s & \leqslant \pi \frac{\psi_{\delta L}(v(\delta), \delta)}{\delta} \\
& \leqslant C(L) \int_{\delta L}^{\varepsilon} \frac{s}{(v(\delta)-v(s))^{2}+(\delta+s)^{2}} \mathrm{~d} s,
\end{aligned}
$$

where $C(L) \downarrow 1$ as $L \rightarrow \infty$. Since

$$
v(s)-v(\delta)=s^{P}-\frac{1}{L^{p}}(\delta L)^{p}>s^{p}\left(1-\frac{1}{L^{p}}\right) \text { for } s \in(\delta L, \varepsilon),
$$

it follows that the integrands from (B.8) are bounded above by the integrable function $\left(1-L^{-p}\right)^{-2} s^{1-2 p}$. A second application of the dominated convergence theorem gives for any $L>1$

$$
\begin{aligned}
\frac{1}{\pi} \int_{0}^{\delta} \frac{s}{v(s)^{2}+s^{2}} \mathrm{~d} s \leqslant \lim _{\delta \downarrow 0} \inf & \frac{\psi_{\delta L}(v(\delta), \delta)}{\delta} \\
& \leqslant \lim _{\delta \downarrow 0} \sup \frac{\psi_{\delta L}(v(\delta), \delta)}{\delta} \leqslant \frac{C(L)}{\pi} \int_{0}^{\delta} \frac{s}{v(s)^{2}+s^{2}} \mathrm{~d} s .
\end{aligned}
$$

Finally we let $L \rightarrow \infty$ in (B.9) and combine the result with (B.7). In terms of the original variables $\left(u(x)=x^{m}, m>1\right)$ we obtain

$$
\lim _{x \downarrow 0} \frac{\psi(x, u(x))}{u(x)}=\frac{1}{2} \int_{0}^{1} \frac{\sin (\pi s)}{\cosh \left(\pi s^{1 / m}\right)-\cos (\pi s)} \mathrm{d} s<\infty .
$$

A similar argument for $m=1$ gives

$$
\lim _{x \downarrow 0} \frac{\psi(x, u(x))}{u(x)}=\infty
$$

that is, the speed of propagation is infinite.

Thus if the interface is given by (B.1) with $m>1$ the toe $S_{1}$ will begin to move at the finite speed given by (B.10). In a similar way one finds that the top $S_{2}$, which is such that $u^{\prime}\left(S_{2}^{-}\right)=m>0$ (compare with the case in which $m=1$ for the toe $S_{1}$ ), will start moving with infinite speed. For the situation at the top, we expect in fact a smoothing of the interface such that, for every $t>0$, $\partial u\left(S_{2}(t), t\right) / \partial x=0$ and finite speed of propagation of the point $S_{2}$ occurs. 